\title{
Adaptive Sensing with Reliable Guarantee under White Gaussian Noise Channels of Sensor Networks
}

\author{
Jun Long, ${ }^{1,2}$ An He, ${ }^{1,3}$ Anfeng Liu, ${ }^{1}$ and Xue Chen ${ }^{1}$ \\ ${ }^{1}$ School of Information Science and Engineering, Central South University, Changsha 410083, China \\ ${ }^{2}$ School of Electronics Engineering and Computer Science, Peking University, Beijing 100871, China \\ ${ }^{3}$ School of Computer and Information Engineering, Central South University of Forestry and Technology, Changsha 410083, China \\ Correspondence should be addressed to Anfeng Liu; afengliu@mail.csu.edu.cn
}

Received 17 May 2015; Accepted 16 June 2015

Academic Editor: Jian-Nong Cao

Copyright (C) 2015 Jun Long et al. This is an open access article distributed under the Creative Commons Attribution License, which permits unrestricted use, distribution, and reproduction in any medium, provided the original work is properly cited.

Quality of sensing is a fundamental research topic in sensor networks. In this paper, we propose an adaptive sensing technique to guarantee the end-to-end reliability while maximizing the lifetime of sensor networks under additive white Gaussian noise channels. First, we conduct theoretical analysis to obtain optimal node number $N^{*}$, node placement $d^{*}$, and node transmission structure $P^{*}$ under minimum total energy consumption and minimum unit data transmission energy consumption. Then, because sensor nodes closer to the sink consume more energy, nodes far from the sink have more residual energy. Based on this observation, we propose an adaptive sensing technique to achieve balanced network energy consumption. It adopts lower reliability requirement and shorter transmission distance for nodes near the sink and adopts higher reliability requirement and farther transmission distance for nodes far from the sink. Theoretical analysis and experimental results show that our design can improve the network lifetime by several times (1-5 times) and network utility by $20 \%$ and the desired reliability level is also guaranteed.

\section{Introduction}

Wireless sensor networks are commonly used for environmental monitoring, surveillance operations, and home or industrial automation [1-3]. Sensor nodes can monitor physical phenomena and can be applied into military, industry scenarios, and daily life, which has attracted wide attention [4-6].

In particular, it is well known that designing energy efficient protocol is the most critical factor for sensor networks $[7,8]$; since sensor nodes are of typically small physical size and limited energy powered by batteries, it is very infeasible to replace or recharge them after deployment because conditions may be unfriendly, or simply it would be too costly $[9,10]$. In recent years, a growing interest in various sensor networks applications is requiring new design factors [11, 12], such as higher reliability [13-15], as well as energy efficiency. Since sensor nodes are of simple structure and low transmission power and are in the complex environment, there has been a certain packet loss rate in the data transmission. In applications such as telemedicine, high packet loss rate may lead to disastrous consequences. How to guarantee the reliable data transmission is an important research topic. There are quite a few studies which have proposed guaranteeing reliable data transmission strategies of sensor network. But the high reliable data transmission can cause a large energy consumption of nodes and thus decrease the network lifetime $[16,17]$. It is conflicting to ensure the quality of sensing while maximizing the lifetime of sensor networks $[18,19]$.

One method for researchers to deal with this conflict is to design an adaptive sensing approach [20] to increase the lifetime and the quality of sensing. Adaptive sensing, which is concerned with how to adaptively determine the sensing time and sensing frequency of sensor nodes by taking into account the quality of sensing requirement, remaining energy, and sensor coordination, has been proven to be an efficient approach to quality of sensing in traditional sensor networks [21-25].

In this paper, we propose an adaptive sensing range optimal approach to guarantee the end-to-end reliability while maximizing the lifetime of sensor networks; the contributions of this paper are as follows. 
(1) We conduct theoretical analysis that there exists optimal node number $N^{*}$, node placement $d^{*}$, and node transmission structure $P^{*}$ which can achieve minimum total energy consumption for data collection and minimum unit data transmitting energy consumption.

The optimization goal can be converted to the following restrictions: optimization for node number $N^{*}$, node placement $d^{*}$, and node transmission structure $P^{*}$. For different networks and applications, we formulate network configuration for optimal utilization efficiency as a multivariety nonlinear optimization problem by jointly optimizing sensor placement, transmission structure, and deployed node number under desired level of reliability; the solving methods are also given in this paper.

(2) We propose an adaptive sensing range optimal approach which can maximize utilization efficiency and give the solvability conditions from mathematics.

The innovation of this paper is that we can further dramatically improve network lifetime through adaptive sensing range optimal design in the case without increasing network cost. Based on the network energy consumption feature that energy consumption is high in regions near the sink but is low in regions far from the sink, we take two measures to improve network lifetime. First, decrease node transmission distance for nodes near the sink and increase it for nodes far from the sink, to balance node energy consumption in the network. Secondly, adopt lower reliability requirement for nodes near the sink but higher reliability requirement for nodes far from the sink, to decrease node energy consumption in regions near the sink and improve the node reliability for nodes far from the sink, although the energy consumption is increased in faraway regions, since they have remaining energy, which can improve network lifetime under desired level of reliability.

(3) Through our extensive theoretical analysis and simulation study, we demonstrate that, for our design, both utilization performance and reliability can be achieved simultaneously. We also demonstrate that our optimal design is superior to previous studies from either total energy consumption, unit data transmitting energy consumption, or network utilization performance.

The rest of this paper is organized as follows. In Section 2, the related works are reviewed. The system model and problem statement are described in Section 3. In Section 4, optimizations for different networks and channel are presented. Section 5 is the analysis and comparison of experimental results. We conclude in Section 6.

\section{Related Work}

How to prolong the network lifetime as well as improve the performance of the network is an important issue in wireless sensor networks. In order to prolong the network lifetime, several optimization measures have been proposed; for example, Zhang et al. [21] show that by optimizing network parameters the network performance can be significantly improved. These parameters include deployed node number $N^{*}$, node placement $d^{*}$, and node transmission structure $P^{*}$. We can optimize these parameters from the physical layer and also can optimize them from network layer, or process cross layer optimization from both physical layer and network layer. Some research analyzes the optimal transmission range from physical layer. Chen et al. [22] define the optimal one-hop length for multihop communications that minimizes the total energy consumption and analyzes the influence of channel parameters on this optimal transmission range in a linear network. The same issue is studied in [23] with a Bit-Meter-perJoule metric where the authors analyze the effects of the network topology, the node density, and the transceiver characteristics on the overall energy expenditure. There is also some research on utility based optimization. Chen et al. [24] introduce a performance measure of utilization efficiency defined as network lifetime per unit deployment cost. In addition to the abovementioned lifetime-utility tradeoff, there exists another tradeoff between network lifetime and the end-toend delay, which is considered separately in literature [25].

In this paper, an adaptive sensing range optimal approach is proposed for single-source linear network, multisource linear network, and grid network under additive white Gaussian noise channels, which not only achieves an optimal utility but also guarantees the end-to-end reliability.

\section{The System Model and Problem Statement}

3.1. Energy Consumption Model. The energy consumption model in this paper is the same as [24]. According to [24], the energy consumption $E_{p}$ for the transmission of one packet is composed of three parts: the energy consumed by the transmitter $E_{t}$, by the receiver $E_{r}$, and by the acknowledgement packet exchange $E_{\mathrm{ACK}}$ :

$$
E_{p}=E_{t}+E_{r}+E_{\mathrm{ACK}} .
$$

The energy model for transmitters and receivers [26] is given, respectively, by

$$
\begin{aligned}
& E_{t}=T_{\text {start }} \cdot P_{\text {start }}+\frac{N_{\text {head }}+N_{b}}{R_{b} \cdot R_{\text {code }}} \cdot\left(P_{\text {txElec }}+\beta_{\text {amp }} \cdot P_{t}\right), \\
& E_{r}=T_{\text {start }} \cdot P_{\text {start }}+\frac{N_{\text {head }}+N_{b}}{R_{b} \cdot R_{\text {code }}} \cdot P_{\text {txElec }},
\end{aligned}
$$

where $P_{t}$ is the transmission power, $N_{\text {head }}$ is the number of bits in the overhead of a packet for the synchronization of physical layer, and $R_{\text {code }}$ is the code rate. The other parameters are described in Table 1.

The energy expenditure model of an acknowledgment is given by

$$
E_{\mathrm{ACK}}=\tau_{\mathrm{ack}} \cdot\left(E_{t}+E_{r}\right),
$$

while

$$
\tau_{\text {ack }}=\frac{N_{\text {ack }}+N_{\text {head }}}{N_{b}+N_{\text {head }}} .
$$

As in [21], the energy model for each bit is

$$
E_{b}=\frac{E_{p}}{N_{b}}=E_{c}+K_{1} \cdot P_{t},
$$


TABLE 1: Some parameters of the transceiver energy consumption [21].

\begin{tabular}{lcc}
\hline Symbol & Description & Value \\
\hline$\alpha$ & Path-loss exponent $(\geq 2)$ & 3 \\
$\beta_{\text {amp }}$ & Amplifier proportional offset $(>1)$ & 14.0 \\
$\tau_{\text {ack }}$ & ACK ratio & 0.08125 \\
$B$ & Bandwidth of channel & $250 \mathrm{kHz}$ \\
$f_{c}$ & Carrier frequency & $2.4 \mathrm{GHz}$ \\
$\lambda$ & Wavelength & \\
$G_{\text {Tant }}$ & Transmitter antenna gain & 1 \\
$G_{\text {Rant }}$ & Receiver antenna gain & 1 \\
$L$ & Circuitry loss ( $\geq 1)$ & 1 \\
$N_{b}$ & Number of bits per packet & 2560 \\
$N_{\text {head }}$ & Number of bits of overhead in a packet & 0 \\
$N_{0}$ & Noise level & $-150 \mathrm{dBm} / \mathrm{Hz}$ \\
$P_{\text {start }}$ & Startup power & $38.7 \mathrm{mV}$ \\
$P_{\text {txElec }}$ & Transmitter circuitry power & $59.1 \mathrm{mV}$ \\
$P_{\text {rxElec }}$ & Receiver circuitry power & $59.1 \mathrm{mV}$ \\
$R_{b}$ & Transmission bit rate & $250 \mathrm{Kbps}$ \\
$T_{\text {start }}$ & Startup time & $0 \mu \mathrm{s}$ \\
$T_{\text {ACK }}$ & ACK duration & $1 \mathrm{~ms}$ \\
\hline & &
\end{tabular}

where $E_{b}, E_{c}$, and $k_{1} \cdot P_{t}$ are, respectively, the total, the constant, and the variable energy consumption per bit. Putting (1)-(3) into (5), we get

$$
\begin{aligned}
E_{c} & =\left(1+\tau_{\text {ack }}\right) \\
\cdot\left(\frac{2 T_{\text {start }} \cdot P_{\text {start }}}{N_{b}}+\left(1+\tau_{\text {head }}\right) \frac{P_{\text {txElec }}+P_{\text {rx Elec }}}{R_{b} R_{\text {code }}}\right), & \\
K_{1} & =\left(1+\tau_{\text {ack }}\right)\left(1+\tau_{\text {head }}\right) \frac{\beta_{\text {amp }}}{R_{b} R_{\text {code }}},
\end{aligned}
$$

where $\tau_{\text {head }}=N_{\text {head }} / N_{b}$.

3.2. Realistic Unreliable Link Model. The realistic unreliable link model is also the same as $[21,27]$. The unreliable radio link probability $(p l)$ is defined using the Packet Error Rate (PER) [21]:

$$
\operatorname{pl}\left(\gamma_{x, x^{\prime}}\right)=1-\operatorname{PER}\left(\gamma_{x, x^{\prime}}\right),
$$

where $\operatorname{PER}(\gamma)$ is the PER obtained from a signal-to-noise ratio (SNR) $\gamma$. And $\gamma_{x, x^{\prime}}$ is usually defined as in [21]

$$
\gamma_{x, x^{\prime}}=K_{2} \cdot P_{t} \cdot d_{\mathrm{hop}}^{-\alpha}
$$

with

$$
K_{2}=\frac{G_{\mathrm{Tant}} \cdot G_{\mathrm{Rant}} \cdot \lambda^{2}}{(4 \pi)^{2} \cdot N_{0} \cdot R_{s} \cdot L},
$$

where $d_{\text {hop }}$ is the distance between nodes $x$ and $x^{\prime}, \lambda$ is the wavelength, and $R_{s}$ is the symbol rate. Other parameters are presented in Table 1 . Note that $R_{b}=R_{s} \cdot b$, where $b$ is the modulation order. The unreliable link models are approximated for AWGN channels, respectively, as follows [24].
For AWGN channels,

$$
\begin{array}{r}
p l_{g}(\gamma)=\left(1-0.1826 \alpha_{m} \cdot \exp \left(-0.5415 \beta_{m} \cdot \gamma\right)\right)^{N_{b}} \\
\left(\beta_{m} \cdot \gamma \geq 2\right) .
\end{array}
$$

As can be seen from the above reliability nature, the link reliability is inversely proportional to the distance between nodes and is proportional to the transmission power; that is, for nodes at link ends, farther distance means lower reliability, but with higher node transmission power, the reliability will be higher.

3.3. Problem Statements. (1) We define the total energy consumption for transmitting one-bit data to the sink as $E_{t}^{\mathrm{AWGN}}$.

(2) We define energy consumption rate $\xi$ as transmitting one-bit data to the sink with energy consumption $E_{t}^{\text {AWGN }}$ divided by the number of nodes $(n)$ participating in transmission; that is,

$$
\xi=\frac{E_{t}^{\mathrm{AWGN}}}{n} .
$$

(3) We define network lifetime $\ell$ as the average amount of time until any sensor runs out of energy (the first failure) $[24,28]$. We define utilization efficiency $\eta$ as network lifetime $\ell$ divided by the number of deployed sensors $N$; that is,

$$
\eta=\frac{\ell}{N}
$$

Utilization efficiency $\eta$ indicates the rate at which network lifetime $\ell$ increases with the number of nodes $N$. It captures the tradeoff between network lifetime and deployment cost.

Our design goal is to find the optimal number of nodes $N^{*}$, sensor placement $d^{*}$, and transmission structure $P^{*}$ that minimize $E_{t}^{\mathrm{AWGN}}$ and $\xi$, while maximizing utilization efficiency $\eta$; that is,

$$
\left\{N^{*}, d^{*}, P^{*}\right\}=\arg \left\{\min _{N, d, P}\left(E_{t}^{\mathrm{AWGN}}, \xi\right), \max _{N, d, P}(\eta)\right\} .
$$

Although the optimization goal of (13) is classified into three categories, that is, $N^{*}, d^{*}$, and $P^{*}$, in the actual network, optimization factors are often complex and frequently changing. In certain applications, all of these three factors are variable; for instance, in a linear network, the number of nodes $N^{*}$, the node deployed position $d^{*}$, and the node transmission structure $P^{*}$ all can be optimized. However, in many applications, certain factors may have been determined, and thus the problem is transformed into an optimization problem under certain restrictions; for instance, in the grid network, the number of deployed nodes $N$ has been determined; thus the optimization goal turns into selecting appropriate placement $d^{*}$, as well as arranging a suitable transmission structure $P^{*}$.

In addition, the optimization goals are interrelated but distinguished from each other; for instance, in a single-source linear network, goals of minimizing $E_{t}^{\mathrm{AWGN}}$ and optimizing 
$\xi$ are not the same; that is, the network configuration which minimizes $E_{t}^{\mathrm{AWGN}}$ does not necessarily minimize $\xi$, since the number of needed nodes is not the minimum when the total energy consumption is the minimum, so $\xi$ is not necessarily the minimum. However, since the node energy consumption for all nodes in the network is the same, the optimal $\xi$ must maximize $\eta$. Meanwhile, in a two-dimensional network, since the node energy consumption is not balanced, the network configuration which optimizes $\xi$ does not necessarily maximize network lifetime, that is, minimizing $\xi$. Therefore, the adaptive sensing range optimization is very complex but critical.

At the same time, the network must ensure the requirement that the end-to-end reliability meets the minimum requirements of the application, such as $C$, as shown in the following formula:

$$
\gamma=\max \left(\prod_{i=1}^{k} \gamma_{i}\right)>C
$$

In summary, the optimization goal of this paper is shown in the following equation:

$$
\begin{aligned}
& \left\{N^{*}, d^{*}, P^{*}\right\}=\arg \left\{\min _{N, d, P}\left(E_{t}^{\mathrm{AWGN}}, \xi\right), \max _{N, d, P}(\eta)\right\} \\
& \text { s.t. } \gamma=\min \left(\prod_{i=1}^{k} \gamma_{i}\right)>C .
\end{aligned}
$$

\section{Scheme Design}

4.1. Single-Source Linear Network. Single-source linear network is shown as the network in Figure 1. In such networks, there is only one node $S_{n}$ which generates data, which is at $D$ from the sink. Since long distance communication has high energy consumption and declines communication reliability sharply, it needs to deploy some node which does not generate data but only forwards data between node $S_{n}$ and the sink, that is, $S_{1}, S_{2}, \ldots S_{n}$. Considering that the node number after optimization is $n$ and the total energy consumption is $E_{t}^{\mathrm{AWGN}}$, then $\xi=E_{t}^{\mathrm{AWGN}} / n$. Since in such networks the energy consumption for each node is the same, the energy consumption for each node is $\xi$. While the network lifetime is $\ell=E_{\text {ini }} / \xi$, where $E_{\text {ini }}$ is the node initial energy, then $\eta=\ell / N=E_{\text {ini }} /(n \xi)=E_{\text {ini }} / E_{t}^{\mathrm{AWGN}}$. That is to say, the optimization goal of minimizing $\xi$ is the same as that of maximizing utilization efficiency $\eta$. Therefore, the optimization in single-source linear network is converted into

$$
\begin{aligned}
& \left\{N^{*}, d^{*}, P^{*}\right\}=\arg \left\{\min _{N, d, P}\left(E_{t}^{\mathrm{AWGN}}, \xi\right)\right\} \\
& \text { s.t. } \gamma=\min \left(\prod_{i=1}^{k} \gamma_{i}\right)>C .
\end{aligned}
$$

The following gives optimization methods for singlesource linear network under different communication channels.

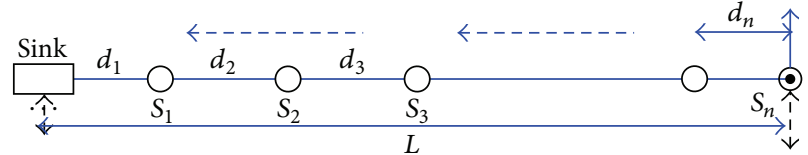

FIGURE 1: Illustration of the line network of only one source node.

Assume the distance between any two nodes is $d_{\text {hop }}$; then data is sent to the sink via $D / d_{\text {hop }}$ hops; to meet reliability $>C$, the following should be ensured:

$$
p l(\gamma)^{D / d_{\text {hop }}} \geq C
$$

In AWGN channels,

$$
p l_{g}(\gamma)=\left(1-0.1826 \alpha_{m} \cdot \exp \left(-0.5415 \beta_{m} \cdot \gamma\right)\right)^{N_{b}} .
$$

Substituted into (17), the following equation can be derived:

$$
\gamma \geq \frac{\ln \left(1-C^{d_{\mathrm{hop}} / N_{b} D}\right)-\ln \left(0.1826 \alpha_{m}\right)}{-0.5415 \beta_{m}} .
$$

Since

$$
\begin{aligned}
\gamma & =K_{2} \cdot P_{t} \cdot d_{\text {hop }}^{-\alpha} \\
K_{2} & =\frac{G_{\text {Tant }} \cdot G_{\text {Rant }} \cdot \lambda^{2}}{(4 \pi)^{2} \cdot N_{0} \cdot R_{s} \cdot L},
\end{aligned}
$$

therefore

$$
P_{t} \geq \frac{d_{\text {hop }}^{\alpha}\left[\ln \left(1-C^{d_{\mathrm{hop}} / N_{b} D}\right)-\ln \left(0.1826 \alpha_{m}\right)\right]}{-0.5415 \beta_{m} K_{2}},
$$

where $\gamma$ is the $\mathrm{S} / \mathrm{N}$ (signal-to-noise ratio), $P_{t}$ is the transmission power, and $p l_{g}(\gamma)$ is the reliability; other parameters can be seen in Table 1 and previous introduction.

Then, the energy consumption for source node sending one-bit data is

$$
\begin{aligned}
E_{b} & =E_{c}+K_{1} \cdot P_{t}, \\
E_{c} & =\left(1+\tau_{\text {ack }}\right) \\
& \cdot\left(\frac{2 T_{\text {start }} \cdot P_{\text {start }}}{N_{b}}+\left(1+\tau_{\text {head }}\right) \cdot \frac{P_{\text {txElec }}+P_{r x \text { Elec }}}{R_{b} \cdot R_{\text {code }}}\right), \\
K_{1} & =\left(1+\tau_{\text {ack }}\right)\left(1+\tau_{\text {head }}\right) \frac{\beta_{\text {amp }}}{R_{b} \cdot R_{\text {code }}} .
\end{aligned}
$$

Thus, the total energy consumption for one-bit source node data to the sink is

$$
\begin{aligned}
& E_{t, 1}^{\mathrm{AWGN}}=\frac{D}{d_{\mathrm{hop}}} \cdot\left(E_{c}\right. \\
& \left.\quad+k_{1} \frac{d_{\mathrm{hop}}^{\alpha}\left[\ln \left(1-C^{d_{\mathrm{hop}} / N_{b} D}\right)-\ln \left(0.1826 \alpha_{m}\right)\right]}{-0.5415 \beta_{m} K_{2}}\right) .
\end{aligned}
$$


In the above optimization goals, $D / d_{\text {hop }}$ is the number of deployed nodes $N$; [21] has proved that the network energy consumption is balanced when the distance between nodes is the same, which achieves highest efficiency. Therefore, in the above optimization goal, only $d_{\text {hop }}$ remains unknown, so the optimization goal is converted into selecting appropriate $d_{\text {hop }}$ to minimize $E_{t, 1}^{\mathrm{AWGN}}$. First, Theorem 1 proves that there must be an optimal $d_{\text {hop }}$ which can minimize network total energy consumption.

Theorem 1. For single-source linear network under AWGN channels, there must be an optimal $d_{\text {hop }}$ which minimizes network total energy consumption $E_{t, 1}^{A W G N}$.

Proof. Consider

$$
\begin{aligned}
& E_{t, 1}^{\mathrm{AWGN}}=\frac{D}{d_{\text {hop }}}\left(E_{c}\right. \\
& \left.\quad+\frac{K_{1} d_{\text {hop }}^{\alpha}\left[\ln \left(1-C^{d_{\text {hop }} / N_{b} D}\right)-\ln \left(0.8126 \alpha_{m}\right)\right]}{-0.5415 \beta_{m} K_{2}}\right) .
\end{aligned}
$$

Namely,

$$
\begin{aligned}
& E_{t, 1}^{\mathrm{AWGN}}=\frac{D}{d_{\mathrm{hop}}} \cdot E_{c} \\
& \quad+\frac{D \cdot K_{1} \cdot d_{\mathrm{hop}}^{\alpha-1}\left[\ln \left(1-C^{d_{\mathrm{hop}} / N_{b} D}\right)-\ln \left(0.8126 \alpha_{m}\right)\right]}{-0.5415 \beta_{m} K_{2}} .
\end{aligned}
$$

The following proves that there exists $d_{\text {hop }}$ which achieves minimum $E_{t, 1}^{\mathrm{AWGN}}$. As can be seen from (25), since $d_{\mathrm{hop}} \epsilon$ $(0, D]$, when $d_{\text {hop }} \rightarrow 0,1 / d_{\text {hop }} \rightarrow+\infty$; thus, $D E_{c} / d_{\text {hop }} \rightarrow$ $+\infty$; at the same time, $\left(D \cdot K_{1} \cdot d_{\text {hop }}^{\alpha-1}\left[\ln \left(1-C^{d_{\text {hop }} / N_{b} D}\right)-\right.\right.$ $\left.\left.\ln \left(0.8126 \alpha_{m}\right)\right]\right) /-0.5415 \beta_{m} K_{2} \rightarrow 0$ (see the proof below), so $E_{t, 1}^{\mathrm{AWGN}} \rightarrow+\infty$. Meanwhile, when $d_{\text {hop }}=D$, we can get $E_{t, 1}^{\mathrm{AWGN}}=E_{c}+K_{1} D^{\alpha}\left[\ln \left(1-C^{1 / N_{b}}\right)-\ln \left(0.8126 \alpha_{m}\right)\right] /-$ $0.5415 \beta_{m} K_{2}$, since it is a bounded positive real number, and $E_{t, 1}^{\mathrm{AWGN}}$ is a continuous derivative function in $d_{\mathrm{hop}} \in(0, D]$, so there must be a $d_{\text {hop }}$ which minimizes $E_{t, 1}^{\mathrm{AWGN}}$. In the following we prove $\lim _{d_{\text {hop }} \rightarrow 0}\left(D \cdot K_{1} \cdot d_{\text {hop }}^{\alpha-1}\left[\ln \left(1-C^{d_{\text {hop }} / N_{b} D}\right)-\right.\right.$ $\left.\left.\ln \left(0.8126 \alpha_{m}\right)\right] /-0.5415 \beta_{m} K_{2}\right)=0$.

First we prove $\lim _{d_{\text {hop }} \rightarrow 0} d_{\text {hop }}^{\alpha-1} \cdot \ln \left(1-C^{d_{\text {hop }} / N_{b} D}\right)=0$. Obviously, The left side of the equal sign is a formula type of $0 \cdot \infty$; then we can turn it into type of $\infty / \infty$; namely,

$$
\begin{gathered}
\lim _{d_{\mathrm{hop}} \rightarrow 0} d_{\mathrm{hop}}^{\alpha-1} \cdot \ln \left(1-C^{d_{\mathrm{hop}} / N_{b} D}\right) \\
\quad=\lim _{d_{\mathrm{hop}} \rightarrow 0} \frac{\ln \left(1-C^{d_{\mathrm{hop}} / N_{b} D}\right)}{1 / d_{\mathrm{hop}}^{\alpha-1}} .
\end{gathered}
$$

According to Table 1, $\alpha=3$; then we can substitute it into (26) and use L' Hospital rule for the first time; then

$$
\begin{gathered}
\lim _{d_{\mathrm{hop}} \rightarrow 0} \frac{\ln \left(1-C^{d_{\mathrm{hop}} / N_{b} D}\right)}{1 / d_{\mathrm{hop}}^{\alpha-1}} \\
=\lim _{d_{\mathrm{hop}} \rightarrow 0} \frac{d_{\mathrm{hop}}^{3} \cdot C^{d_{\mathrm{hop}} / N_{b} D} \cdot \ln C}{2 N_{b} D\left(1-C^{d_{\mathrm{hop}} / N_{b} D}\right)} .
\end{gathered}
$$

As can be seen, (27) is type of 0/0; then use L' Hospital rule again; that is,

$$
\begin{gathered}
\lim _{d_{\text {hop }} \rightarrow 0} \frac{d_{\text {hop }}^{3} \cdot C^{d_{\text {hop }} / N_{b} D} \cdot \ln C}{2 N_{b} D\left(1-C^{d_{\text {hop }} / N_{b} D}\right)} \\
=\lim _{d_{\text {hop }} \rightarrow 0} \frac{3 d_{\text {hop }}^{2} N_{b} D+d_{\text {hop }}^{3} \ln C}{-2 N_{b} D}=0 .
\end{gathered}
$$

Therefore, $\lim _{d_{\text {hop }} \rightarrow 0} d_{\text {hop }}^{\alpha-1} \cdot \ln \left(1-C^{d_{\text {hop }} / N_{b} D}\right)=0$.

Different from previous studies, Theorem 1 in this paper mathematically strictly proves that there must be an optimal $d_{\text {hop }}$ which maximizes network utilization efficiency. However, since the optimized equation is a higher-order equation, analytical solution cannot be given [24]. But since $D$ is a limited number and the required accuracy of node transmission distance $d_{\text {hop }}$ in actual applications is not particularly high, thus, through exhaustive search method, a solution which meets applications' requirement is very easy to get.

Figure 2 shows the energy consumption under $D=$ $500 \mathrm{~m}, C=0.9$, and different $d_{\text {hop }}$; as can be seen, the energy consumption curve is a concave, and thus there is a minimum extreme point; namely, when $d_{\text {hop }}=56.156 \mathrm{~m}$, it obtains the optimal value.

4.2. Multisource Linear Network. In the actual network, the common linear network is where each node in the network is deployed to monitor the surrounding environment and generates sensed data; such linear networks are widely applied into applications such as roads, oil pipelines, and border detection. Since any one route in the widely used two-dimensional network can also be considered as a linear network, its research has important significance, which is referred to as a multisource linear network in this paper. As shown in Figure 3, there are $n$ nodes linearly deployed in the network; each node generates one data packet in one data collection round and transmits its data packets to the sink. For node $S_{1}$ nearest to the sink, the data load is $n$ data packets, and for node $S_{2}$, it is $n-1$ data packets and so forth. For node $S_{n}$, the data load is one data packet.

This section focuses on optimization for a multisource linear network; there are three optimization goals; they are $\min _{N, d, P}\left(E_{\text {tot }}, \xi\right), \max _{N, d, P}(\eta)$.

First, we prove that the network total energy consumption is the minimum when $n$ nodes are equidistantly deployed in a multisource linear network. As shown in Theorem 2, we give the energy consumption for each node in the network. 


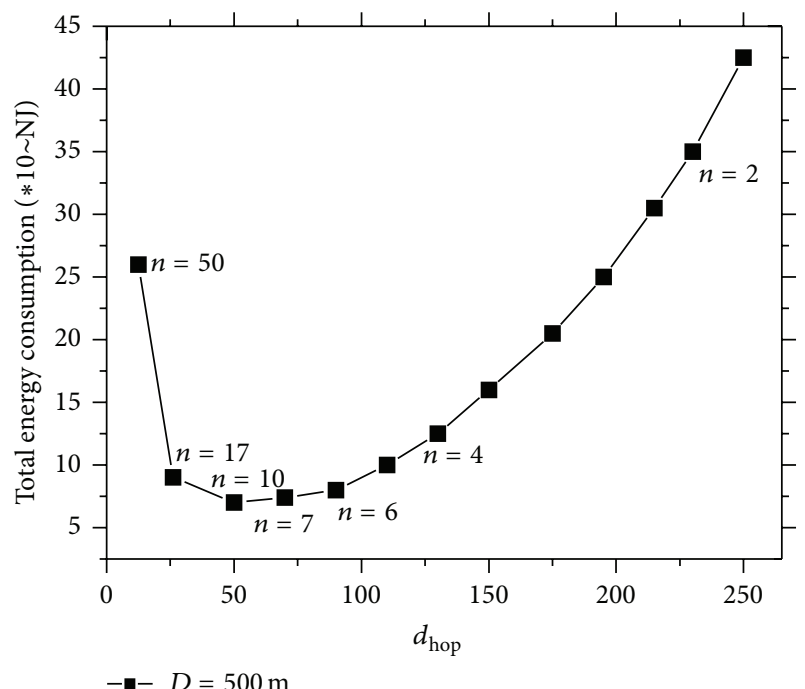

Figure 2: Network total energy consumption under AWGN channels.

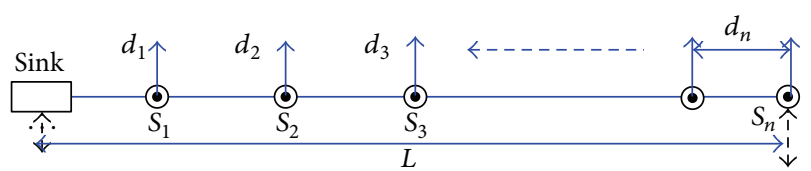

FIGURE 3: Illustration of the line network of each node as source.

Theorem 2. Assume the number of equidistantly deployed nodes is $n$; then the energy consumption for node $i$ is

$$
E_{i, 2}=(n-i+1) \varsigma\left(E_{c}+K_{1} \cdot P_{t, 2}\right)
$$

where $P_{t, 2}=d_{h o p}^{\alpha}\left[\ln \left(1-C^{1 / n N_{b}}\right)-\ln \left(0.1826 \alpha_{m}\right)\right] /-$ $0.5415 \beta_{m} K_{2}$.

Proof. Obviously, in the network as shown in Figure 3, the data load of node $i$ includes data of its own and data packets of nodes afterwards, namely, $(n-i+1)$.

Assume the number of bits for each data packet is $\varsigma$; then the data load is $(n-i+1) \varsigma$.

According to (24), we get

$$
P_{t, 2}=\frac{d_{\text {hop }}^{\alpha}\left[\ln \left(1-C^{1 / n N_{b}}\right)-\ln \left(0.1826 \alpha_{m}\right)\right]}{-0.5415 \beta_{m} K_{2}} .
$$

According to (25),

$$
E_{i, 2}=(n-i+1) \varsigma\left(E_{c}+K_{1} \cdot P_{t, 2}\right) .
$$

Theorem 3. Under AWGN channels, for a linear network whose length is $D$, there are $n$ nodes, and each node generates one data packet in one data collection round and sends it to the sink; then the network total energy consumption is the minimum when these nodes are equidistantly deployed.
Proof. Theorem 2 proves that, for node $i$ in $n$ equidistantly deployed nodes, the energy consumption is

$$
\begin{aligned}
& E_{i, 2}^{\mathrm{AWGN}}=(n-i+1) \varsigma\left(E_{c}+K_{1} \cdot P_{t, 2}^{\mathrm{AWGN}}\right)=\left(E_{c}\right. \\
& \left.\quad+K_{1} \cdot \frac{d_{i}^{\alpha}\left[\ln \left(1-C^{1 / N_{b} n}\right)-\ln \left(0.8126 \alpha_{m}\right)\right]}{-0.5415 \beta_{m} K_{2}}\right)(n \\
& \quad+1-i)
\end{aligned}
$$

Then the total energy consumption is

$$
\begin{aligned}
& E_{t, 2}^{\mathrm{AWGN}}=\sum_{i=1}^{n} E_{i, 2}=\left(E_{c}+K_{1}\right. \\
& \left.\cdot \frac{d_{\text {hop }}^{\alpha}\left[\ln \left(1-C^{1 / N_{b} n}\right)-\ln \left(0.8126 \alpha_{m}\right)\right]}{-0.5415 \beta_{m} K_{2}}\right) \\
& \cdot\left(\frac{(n+1) n}{2}\right)
\end{aligned}
$$

subject to $n d_{\text {hop }}=D$.

If it is not equidistant, set the distance of node $i$ as $d_{i}$; then the total energy consumption is

$$
\begin{aligned}
& E_{\Delta d}=\sum_{i=1}^{n}\left(\left(E_{c}+K_{1}\right.\right. \\
& \left.\quad \cdot \frac{d_{i}^{\alpha}\left[\ln \left(1-C^{1 / N_{b} n}\right)-\ln \left(0.8126 \alpha_{m}\right)\right]}{-0.5415 \beta_{m} K_{2}}\right)(n+1 \\
& -i))
\end{aligned}
$$

subject to $d_{1}+d_{2}+\cdots+d_{n}=D$.

Then, we only need to prove $E_{t, 2}^{\mathrm{AWGN}} \leq E_{\Delta d}$, that is, to prove

$$
\begin{aligned}
& \left(\frac{(n+1) n}{2}\right) E_{c}+\sum_{i=1}^{n} K_{1} \\
& \cdot \frac{d_{i}^{\alpha}\left[\ln \left(1-C^{1 / N_{b} n}\right)-\ln \left(0.8126 \alpha_{m}\right)\right]}{-0.5415 \beta_{m} K_{2}}(n+1-i) \\
& \geq\left(\frac{(n+1) n}{2}\right) E_{c}+K_{1} \\
& \quad \cdot \frac{d_{\text {hop }}^{\alpha}\left[\ln \left(1-C^{1 / N_{b} n}\right)-\ln \left(0.8126 \alpha_{m}\right)\right]}{-0.5415 \beta_{m} K_{2}}\left(\frac{(n+1) n}{2}\right) .
\end{aligned}
$$


We need to prove

$$
\begin{aligned}
K_{1} & \cdot \frac{\left[\ln \left(1-C^{1 / N_{b} n}\right)-\ln \left(0.8126 \alpha_{m}\right)\right]}{-0.5415 \beta_{m} K_{2}} \\
& \cdot \sum_{i=1}^{n} d_{i}^{\alpha}(n+1-i) \geq K_{1} \\
& \cdot \frac{\left[\ln \left(1-C^{1 / N_{b} n}\right)-\ln \left(0.8126 \alpha_{m}\right)\right]}{-0.5415 \beta_{m} K_{2}}\left(\frac{(n+1) n}{2}\right) \\
& \cdot d_{\text {hop }}^{\alpha} .
\end{aligned}
$$

From the above, we can get

$$
\sum_{i=1}^{n} d_{i}^{\alpha}(n+1-i) \geq\left(\frac{(n+1) n}{2}\right) d_{\mathrm{hop}}^{\alpha}
$$

In the following we prove (37):

$$
\text { Min } E_{t}^{\mathrm{AWGN}}=\sum_{i=1}^{n} d_{i}^{\alpha}(n+1-i),
$$

Subject to: $d_{1}+d_{2}+\cdots+d_{n}=D$.

Set $F=E_{1}+E_{2}+\cdots+E_{n}+\lambda\left(d_{1}+d_{2}+\cdots+d_{n}-D\right)$, where $\lambda \neq 0$ is Lagrange multiplier. According to Lagrange multipliers

$$
\begin{gathered}
\frac{\partial E_{1}}{\partial d_{1}}+\lambda=0 \\
\vdots \\
\frac{\partial E_{n}}{\partial d_{n}}+\lambda=0 \\
d_{1}+d_{2}+\cdots+d_{n}=D .
\end{gathered}
$$

Equation (39) shows that when $\partial E_{1} / \partial d_{1}=\cdots=\partial E_{n} / \partial d_{n}=$ $-\lambda, F$ can obtain the minimum value; since all nodes are the same, $E_{i}=d_{i}^{\alpha}(n+1-i)$ in linear network.

Since $\partial^{2} E_{i} / \partial d_{i}^{2}=(n+1-i) \cdot \alpha \cdot(\alpha-1) d_{i}^{\alpha-2}>0$, then we can know that when $\alpha>2$, if the above formula is bigger than 0 , then $\partial E / \partial d$ is a function with $d$ which is strictly monotonically increasing, and then (39) is solvable; $d_{1}=d_{2}=\cdots=d_{n}=D / n$. Thus, Min $E_{t}^{\mathrm{AWGN}}=((n+$ 1) $n / 2) d_{\text {hop }}^{\alpha}\left(d_{\text {hop }}=D / n\right)$.

Therefore, (37) is proved.

Theorems 2 and 3 show the total energy consumption is the minimum when nodes are equidistantly deployed. Therefore, this paper proves theoretically that if the number of deployed nodes is $n$, then the optimization of node placement $d^{*}$ is deploying these $n$ nodes equidistantly in a multisource linear network. Then, the following question is to determine the number $n$ to minimize network total energy consumption; that is, $\min \left(E_{t, 2}^{\mathrm{AWGN}}\right)=\min \left(\sum_{i=1}^{n} E_{i, 2}\right)$.
Theorem 4. For multisource linear network under AWGN channels, there must be an optimal $d_{\text {hop }}$, which can minimize $E_{t, 2}^{A W G N}$ in $(0, D]$.

Proof. Consider

$$
\begin{aligned}
& E_{t, 2}^{\mathrm{AWGN}}=\left(E_{c}+K_{1}\right. \\
& \left.\cdot \frac{d_{\text {hop }}^{\alpha}\left[\ln \left(1-C^{1 / N_{b} n}\right)-\ln \left(0.8126 \alpha_{m}\right)\right]}{-0.5415 \beta_{m} K_{2}}\right) \\
& \cdot\left(\frac{(n+1) n}{2}\right) .
\end{aligned}
$$

Substitute $n=D / d_{\text {hop }}$ into the above formula; that is,

$$
\begin{aligned}
& E_{t, 2}^{\mathrm{AWGN}}=\left(E_{c}+K_{1}\right. \\
& \left.\cdot \frac{d_{\mathrm{hop}}^{\alpha}\left[\ln \left(1-C^{d_{\mathrm{hop}} / N_{b} D}\right)-\ln \left(0.8126 \alpha_{m}\right)\right]}{-0.5415 \beta_{m} K_{2}}\right) \\
& \cdot\left(\frac{\left(D / d_{\mathrm{hop}}+1\right)\left(D / d_{\mathrm{hop}}\right)}{2}\right) .
\end{aligned}
$$

Substitute $\alpha=3$ into the above formula; then

$$
\begin{aligned}
& E_{t, 2}^{\mathrm{AWGN}} \\
& =E_{c}\left(\frac{D^{2}}{2 d_{\text {hop }}^{2}}+\frac{D}{2 d_{\text {hop }}}\right) \\
& \quad+\frac{K_{1} D^{2} d_{\text {hop }}\left[\ln \left(1-C^{d_{\text {hop }} / N_{b} D}\right)-\ln \left(0.8126 \alpha_{m}\right)\right]}{-0.5415 \beta_{m} K_{2} \cdot 2} \\
& \quad+\frac{K_{1} D d_{\text {hop }}^{2}\left[\ln \left(1-C^{d_{\text {hop }} / N_{b} D}\right)-\ln \left(0.8126 \alpha_{m}\right)\right]}{-0.5415 \beta_{m} K_{2} \cdot 2} .
\end{aligned}
$$

When $d_{\text {hop }} \rightarrow 0$, obviously, $E_{c}\left(D^{2} / 2 d_{\text {hop }}^{2}+D / 2 d_{\text {hop }}\right) \rightarrow$ $+\infty$, and we can know from the above that

$$
\begin{aligned}
& \frac{K_{1} D d_{\text {hop }}^{2}\left[\ln \left(1-C^{d_{\text {hop }} / N_{b} D}\right)-\ln \left(0.8126 \alpha_{m}\right)\right]}{-0.5415 \beta_{m} K_{2} \cdot 2} \\
& \longrightarrow 0, \\
& \frac{K_{1} D^{2} d_{\text {hop }}\left[\ln \left(1-C^{d_{\text {hop }} / N_{b} D}\right)-\ln \left(0.8126 \alpha_{m}\right)\right]}{-0.5415 \beta_{m} K_{2} \cdot 2} \\
& \longrightarrow 0
\end{aligned}
$$

(see the following proof). 
Thus, when $d_{\text {hop }} \rightarrow 0, E_{t, 2}^{\mathrm{AWGN}} \rightarrow+\infty$, while when $d=$ $D$, we can get

$$
\begin{aligned}
E_{t, 2}^{\mathrm{AWGN}}=\left(E_{c}+K_{1}\right. \\
\left.\cdot \frac{D^{\alpha}\left[\ln \left(1-C^{1 / N_{b}}\right)-\ln \left(0.8126 \alpha_{m}\right)\right]}{-0.5415 \beta_{m} K_{2}}\right) .
\end{aligned}
$$

Obviously, $E_{t, 2}^{\mathrm{AWGN}}$ is a bounded positive real number, and it is a continuous derivative function in $(0, D]$; then, there must be one $d_{\text {hop }}$ which can make $E_{t, 2}^{\text {AWGN }}$ obtain the minimum value in $(0, D]$; in the following we prove

$$
\begin{aligned}
& \lim _{d_{\text {hop }} \rightarrow 0} \frac{K_{1} D^{2} d_{\text {hop }}\left[\ln \left(1-C^{d_{\text {hop }} / N_{b} D}\right)-\ln \left(0.8126 \alpha_{m}\right)\right]}{-0.5415 \beta_{m} K_{2} \cdot 2} \\
& \quad=0 .
\end{aligned}
$$

First, we prove

$$
\lim _{d_{\text {hop }} \rightarrow 0} d_{\text {hop }} \ln \left(1-C^{d_{\text {hop }} / N_{b} D}\right)=0 .
$$

Obviously, the left side of the (46) is a type of $0 \cdot \infty$; we can convert it into type of $\infty / \infty$; that is,

$$
\lim _{d_{\text {hop }} \rightarrow 0} \frac{\ln \left(1-C^{d_{\text {hop }} / N_{b} D}\right)}{1 / d_{\text {hop }}} .
$$

Using L' Hospital rule yields

$$
\begin{aligned}
& \lim _{d_{\text {hop }} \rightarrow 0} \frac{\ln \left(1-C^{d_{\text {hop }} / N_{b} D}\right)}{1 / d_{\text {hop }}} \\
& =\lim _{d_{\text {hop }} \rightarrow 0} \frac{\ln C}{N_{B} D} \cdot \frac{d_{\text {hop }}^{2} \cdot C^{d_{\text {hop }} / N_{b} D}}{\left(1-C^{d_{\text {hop }} / N_{b} D}\right)} .
\end{aligned}
$$

Based on analysis, the above formula is a type of $0 / 0$; using L' Hospital rule again yields

$$
\begin{aligned}
& \lim _{d_{\text {hop }} \rightarrow 0} \frac{\ln C}{N_{B} D} \cdot \frac{d_{\text {hop }}^{2} \cdot C^{d_{\text {hop }} / N_{b} D}}{\left(1-C^{d_{\text {hop }} / N_{b} D}\right)} \\
& =\lim _{d_{\text {hop }} \rightarrow 0} \frac{\ln C \cdot d_{\text {hop }}^{2}+2 d_{\text {hop }} N_{B} D}{N_{B} D}=0 .
\end{aligned}
$$

Then (46) is proved, and thus the origin formula is proved.

Theorem 3 proves that when $n$ nodes are equidistantly deployed, the network performance is optimal. Theorem 4 shows that there must be an optimal node distance $d_{\text {hop }}$ which can minimize network energy consumption, and there must be an optimal $n$ which can minimize network consumption. Figure 4 shows the total energy consumption for multisource linear network in AWGN channels under different number of deployed nodes. As can be seen from Figure 4, when $D=$ $500 \mathrm{~m}, n=5$; it obtains the optimal value. In fact, Theorem 4 gives the minimum total energy consumption $E_{t, 2}^{\mathrm{AWGN}}$ for data collection; however, since the number of nodes is different in routing path, according to the definition of energy consumption rate in Section 3.3, the goal is to maximize $\xi$, that is, to minimize unit node energy consumption; thus the energy consumption for unit node is

$$
\begin{aligned}
& E_{p, 2}^{\mathrm{AWGN}}=\frac{E_{t, 2}^{\mathrm{AWGN}}}{n}, \\
& E_{p, 2}^{\mathrm{AWGN}}=\left(E_{c}\right. \\
& \left.\quad+\frac{K_{1} d_{\mathrm{hop}}^{\alpha}\left[\ln \left(1-C^{1 / N_{b} n}\right)-\ln \left(0.8126 \alpha_{m}\right)\right]}{-0.5415 \beta_{m} K_{2}}\right) \\
& \quad \cdot\left(\frac{(n+1)}{2}\right) .
\end{aligned}
$$

Then the optimization goal is to minimize $E_{p, 2}^{\mathrm{AWGN}}$, that is, $\min \left\{E_{p, 2}^{\mathrm{AWGN}}\right\}$.

Theorem 5. Under AWGN channels, for multisource linear network, there exists one $d_{\text {hop }}$ which can minimize $E_{p, 2}^{A W G N}$ in $(0, D]$.

Proof. Consider

$$
\begin{aligned}
& E_{p, 2}^{\mathrm{AWGN}}=\left(E_{c}+K_{1}\right. \\
& \left.\cdot \frac{d_{\mathrm{hop}}^{\alpha}\left[\ln \left(1-C^{1 / N_{b} n}\right)-\ln \left(0.8126 \alpha_{m}\right)\right]}{-0.5415 \beta_{m} K_{2}}\right) \\
& \cdot\left(\frac{(n+1)}{2}\right) .
\end{aligned}
$$

Substitute $n=D / d_{\text {hop }}$ into the above formula; that is,

$$
\begin{aligned}
& E_{p, 2}^{\mathrm{AWGN}}=E_{c}\left(\frac{1}{2}+\frac{D}{2 d_{\text {hop }}}\right) \\
& +\frac{K_{1} D \cdot d_{\text {hop }}^{\alpha-1}\left[\ln \left(1-C^{d_{\text {hop }} / N_{b} D}\right)-\ln \left(0.8126 \alpha_{m}\right)\right]}{-0.5415 \beta_{m} K_{2} \cdot 2} \\
& +\frac{K_{1} d_{\text {hop }}^{\alpha}\left[\ln \left(1-C^{d_{\text {hop }} / N_{b} D}\right)-\ln \left(0.8126 \alpha_{m}\right)\right]}{-0.5415 \beta_{m} K_{2} \cdot 2} .
\end{aligned}
$$

When $d_{\text {hop }} \rightarrow 0$, obviously, $E_{c}\left(1 / 2+D / 2 d_{\text {hop }}\right) \rightarrow+\infty$, as can be seen from the above, yields

$$
\frac{K_{1} D d_{\text {hop }}^{2}\left[\ln \left(1-C^{d_{\text {hop }} / N_{b} D}\right)-\ln \left(0.8126 \alpha_{m}\right)\right]}{-0.5415 \beta_{m} K_{2} \cdot 2}
$$




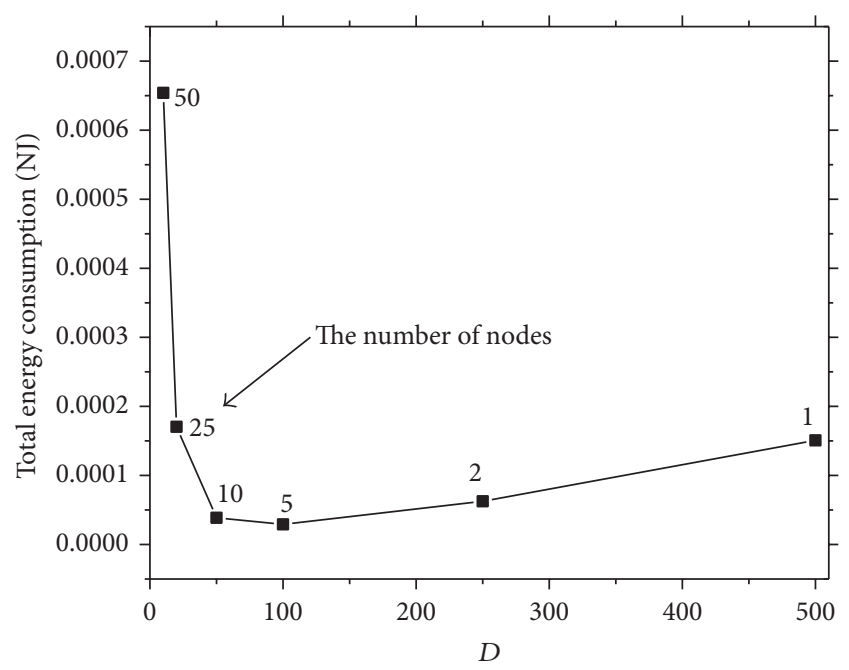

Figure 4: The total energy consumption under different $d_{\text {hop }}$ (AWGN).

Similarly, we can get

$$
\begin{aligned}
& \frac{K_{1} d_{\text {hop }}^{\alpha}\left[\ln \left(1-C^{d_{\text {hop }} / N_{b} D}\right)-\ln \left(0.8126 \alpha_{m}\right)\right]}{-0.5415 \beta_{m} K_{2} \cdot 2} \\
& \longrightarrow+\infty ;
\end{aligned}
$$

thus, when $d_{\text {hop }} \rightarrow 0, E_{p, 2}^{\text {AWGN }} \rightarrow+\infty$. Meanwhile, when $d_{\text {hop }}=D$, we can get

$$
\begin{aligned}
& E_{p, 2}^{\mathrm{AWGN}}=\left(E_{c}+K_{1}\right. \\
&\left.\cdot \frac{D^{\alpha}\left[\ln \left(1-C^{1 / N_{b}}\right)-\ln \left(0.8126 \alpha_{m}\right)\right]}{-0.5415 \beta_{m} K_{2}}\right)
\end{aligned}
$$

Obviously, $E_{p, 2}^{\mathrm{AWGN}}$ is a bounded positive real number, and it is a continuous derivative function in $(0, D]$; then, there must be one $d_{\text {hop }}$ which can make $E_{p, 2}^{\text {AWGN }}$ obtain the minimum value in $(0, D]$.

Figure 5 shows the energy consumption $E_{p, 2}^{\mathrm{AWGN}}$ under different node number $n$, as can be seen; in multisource linear network, there exists an optimal $n$ which can achieve minimum $E_{p, 2}^{\mathrm{AWGN}}$. In summary, we get the following conclusions. (1) When $n$ is determined, if nodes are equidistantly deployed, the network total energy consumption is the minimum, so is the energy consumption per node. (2) When $n$ can be optimized, there must be an optimal $n$ which can minimize network total energy consumption and energy consumption per node. In these two situations, the optimal $n$ is not necessarily the same.

As discussed previously, although energy utilization can be optimized, the network utilization is not necessarily optimized at the same time. The reason is that the network lifetime is determined by the node which has the maximum

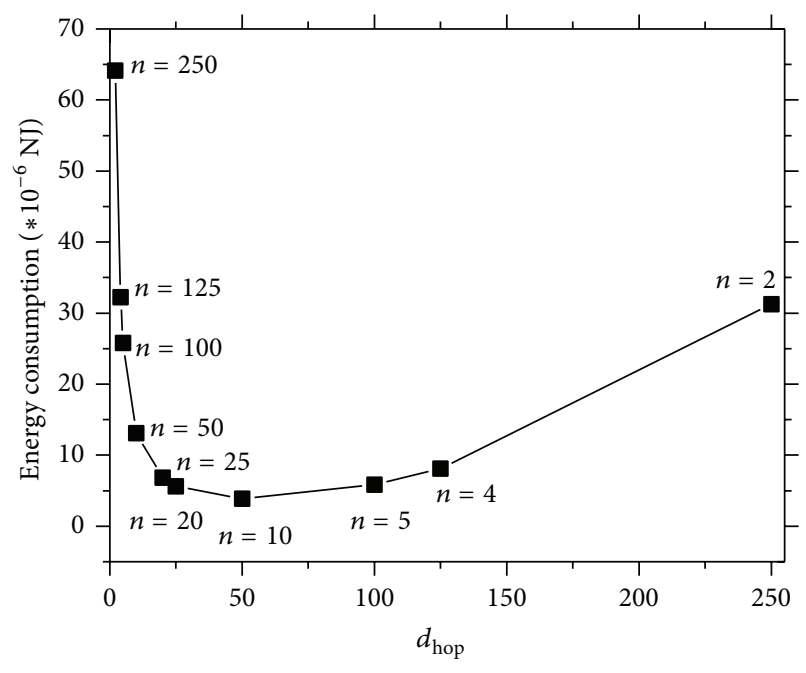

$-\square=500 \mathrm{~m}$

FIGURE 5: The energy consumption per node (AWGN).

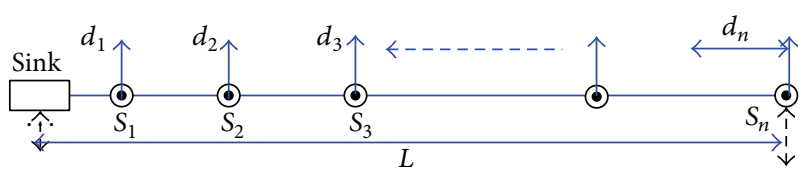

Figure 6: Illustration of the unequidistant linear network.

energy consumption, while in multisource network it is the node which is nearest to the sink. Therefore, the network utilization optimization is to minimize the energy consumption of this node; that is,

$$
\min \max \left(E_{i, 2}\right) \mid i \in\{1, \ldots, n\} .
$$

After previous optimization, the network node number $n$ is determined, so the node data load is determined; then the energy consumption for all other nodes is constant except for $P_{t} ; P_{t}$ is calculated as the following:

$$
P_{t}=-\frac{d_{\text {hop }}^{\alpha}(4 \pi)^{2} N_{0} L R_{S}}{0.5415 \beta_{m} G_{\text {Tant }} G_{\text {Rant }} \lambda^{2}} \ln \frac{1-C^{1 / N_{b}}}{0.1826 \alpha_{m}} .
$$

Obviously, the network utilization optimization is decreasing node energy consumption, or decreasing the transmission distance $d_{\text {hop }}$, or decreasing the reliability $C$.

First, we discuss how to decrease $d_{\text {hop }}$ for nodes near the sink and increase $d_{\text {hop }}$ for nodes far from the sink. As shown in Figure 6, for nodes nearer to the sink, data load is much higher. Thus, we can decrease the transmission distance for these nodes to decrease the energy consumption for unit data transmission.

Then, the question is converted to how to determine $d_{1}, d_{2}, \ldots, d_{n} \mid d_{0} \leq d_{1} \leq d_{2} \leq \cdots \leq d_{n}$, where $d_{1}+d_{2}+$ $\cdots+d_{n}=D$ in Figure 6 , to optimize (56), where the distance $d_{0}>0$ between two nodes must be greater than a specified constant value. 
Theorem 6. For multisource linear network under AWGN channels, solving a set of $d_{1}, d_{2}, \ldots, d_{n}$ to achieve $\min \max \left(E_{i, 2}\right) \mid i \in\{1, \ldots, n\}$, s.t. $d_{1}+d_{2}+\cdots+d_{n}=D$, $d_{0} \leq d_{1} \leq d_{2} \leq \cdots \leq d_{n}$, is to solve the following:

$$
\begin{gathered}
n M_{1} d_{1}^{\alpha}-(n-1) M_{1} d_{2}^{\alpha}+E_{c}=0, \\
(n-1) M_{1} d_{2}^{\alpha}-(n-2) M_{1} d_{3}^{\alpha}+E_{c}=0, \\
\vdots \\
2 M_{1} d_{n-1}^{\alpha}-M_{1} d_{n}^{\alpha}+E_{c}=0, \\
\sum_{i=1}^{n} d_{i}=D, \\
M_{1}=\frac{K_{1}\left[\ln \left(1-C^{1 / n N_{b}}\right)-\ln \left(0.1826 \alpha_{m}\right)\right]}{-0.5415 \beta_{m} K_{2}} .
\end{gathered}
$$

Proof. When the energy consumption for all nodes is balanced, the network lifetime is the maximum, namely, that is to solve how to balance all node energy consumption.

On the other hand, to solve $E_{i}=N_{b}(n+1-i)\left(E_{c}+K_{1}\right.$. $\left.P_{t_{i}}\right) i=1, \ldots, n$, is to solve $E_{i}=E_{j}(i \neq j)$ :

$$
\begin{gathered}
E_{1}=E_{2}, \\
E_{2}=E_{3}, \\
\vdots \\
E_{n-1}=E_{n}, \\
\sum_{i=1}^{n} d_{i}=D .
\end{gathered}
$$

Previous discussion shows that

$$
\begin{aligned}
P_{t_{i}} & =\frac{d_{i}^{\alpha}\left[\ln \left(1-C^{1 / n N_{b}}\right)-\ln \left(0.1826 \alpha_{m}\right)\right]}{-0.5415 \beta_{m} K_{2}}, \\
E_{i}= & N_{b}(n+1-i)\left(E_{c}+K_{1}\right. \\
& \left.\frac{d_{i}^{\alpha}\left[\ln \left(1-C^{1 / n N_{b}}\right)-\ln \left(0.1826 \alpha_{m}\right)\right]}{-0.5415 \beta_{m} K_{2}}\right) .
\end{aligned}
$$

Set

$$
M_{1}=\frac{K_{1}\left[\ln \left(1-C^{1 / n N_{b}}\right)-\ln \left(0.1826 \alpha_{m}\right)\right]}{-0.5415 \beta_{m} K_{2}} .
$$

Substitute the above formula into (60); that is,

$$
E_{i}=N_{b}(n+1-i)\left(E_{c}+M_{1} d_{i}^{\alpha}\right) .
$$

Substituting (60) into (59), after reorganization, we can get (58).
Inference 1. The solvability criterion of (58) is as follows: if $g_{1}(\varepsilon)=\sum_{i=1}^{n}\left(\left(n M_{1} \varepsilon^{\alpha}+(i-1) E_{c}\right) /(n+1-i) M_{1}\right)^{1 / \alpha}-D \leq$ $0 \mid \varepsilon \rightarrow 0$, then (58) is solvable.

Proof. Computing the front $n-1$ equations of (58) yields

$$
d_{i}=\left(\frac{n M_{1} d_{1}^{\alpha}+(i-1) E_{c}}{(n+1-i) M_{1}}\right)^{1 / \alpha} \quad(i \geq 1)
$$

That is, represent all $d_{i}$ with $d_{1}\left(D \geq d_{1}>0\right)$ and then substituting them into the $n$th equation of (59) yields

$$
\sum_{i=1}^{n}\left(\frac{n M_{1} d_{1}^{\alpha}+(i-1) E_{c}}{(n+1-i) M_{1}}\right)^{1 / \alpha}=D
$$

Assume $g_{1}\left(d_{1}\right)=\sum_{i=1}^{n}\left(\left(n M_{1} d_{1}^{\alpha}+(i-1) E_{c}\right) /(n+1-\right.$ i) $\left.M_{1}\right)^{1 / \alpha}-D$; through the analysis, we can know that $g_{1}\left(d_{1}\right)$ is a monotonically increasing function in $d_{1} \in(0, D]$, and, obviously, when $d_{1}=D$, we can get $g_{1}\left(d_{1}\right)>0$. Meanwhile, when $d_{1} \rightarrow 0$, it yields

$$
g_{1}(\varepsilon)=\sum_{i=1}^{n}\left(\frac{n M_{1} \varepsilon^{\alpha}+(i-1) E_{c}}{(n+1-i) M_{1}}\right)^{1 / \alpha}-D \mid \varepsilon \longrightarrow 0
$$

Therefore, only if (65) is not bigger than 0 does the original equation have solutions and we can get the appropriate solution.

We use a specific example to illustrate the application of Theorem 6. That is, the network length $D=500 \mathrm{~m}$; as can be seen from Theorem 5 , the energy consumption per node is the minimum when $n=6$, while, in Theorem 5 , nodes are equidistantly deployed, and thus the energy consumption for each node is not the same. Theorem 6 describes how to optimize the distance between these six nodes, so that the energy consumption of these six nodes is equal to obtain maximum network lifetime and thus optimize $\eta=\ell / N$ in (12).

Since $E_{i}=N_{b}(n+1-i)\left(E_{c}+K_{1} \cdot P_{t_{i}}\right), i=1, \ldots, 6, n=6$, we need $E_{i}=E_{j}(i \neq j)$ and $d_{1}+d_{2}+\cdots+d_{n}=D$; then we can get the following equation set:

$$
\begin{aligned}
E_{1} & =E_{2}, \\
E_{2} & =E_{3}, \\
E_{3} & =E_{4}, \\
E_{4} & =E_{5}, \\
E_{5} & =E_{6}, \\
d_{1}+d_{2}+\cdots+d_{6} & =D .
\end{aligned}
$$


Since there are six equations for six unknown factors, they are solvable; according to (66), we can get

$$
\begin{aligned}
& 6 N_{b}\left(E_{c}+K_{1}\right. \\
& \left.\cdot \frac{d_{1}^{\alpha}\left[\ln \left(1-C^{1 / n N_{b}}\right)-\ln \left(0.1826 \alpha_{m}\right)\right]}{-0.5415 \beta_{m} K_{2}}\right) \\
& =5 N_{b}\left(E_{c}+K_{1}\right. \\
& \left.\cdot \frac{d_{2}^{\alpha}\left[\ln \left(1-C^{1 / n N_{b}}\right)-\ln \left(0.1826 \alpha_{m}\right)\right]}{-0.5415 \beta_{m} K_{2}}\right), \\
& 5 N_{b}\left(E_{c}+K_{1}\right. \\
& \left.\cdot \frac{d_{2}^{\alpha}\left[\ln \left(1-C^{1 / n N_{b}}\right)-\ln \left(0.1826 \alpha_{m}\right)\right]}{-0.5415 \beta_{m} K_{2}}\right) \\
& =4 N_{b}\left(E_{c}+K_{1}\right. \\
& \left.\cdot \frac{d_{3}^{\alpha}\left[\ln \left(1-C^{1 / n N_{b}}\right)-\ln \left(0.1826 \alpha_{m}\right)\right]}{-0.5415 \beta_{m} K_{2}}\right) \text {, } \\
& 4 N_{b}\left(E_{c}+K_{1}\right. \\
& \left.\cdot \frac{d_{3}^{\alpha}\left[\ln \left(1-C^{1 / n N_{b}}\right)-\ln \left(0.1826 \alpha_{m}\right)\right]}{-0.5415 \beta_{m} K_{2}}\right) \\
& =3 N_{b}\left(E_{c}+K_{1}\right. \\
& \left.\cdot \frac{d_{4}^{\alpha}\left[\ln \left(1-C^{1 / n N_{b}}\right)-\ln \left(0.1826 \alpha_{m}\right)\right]}{-0.5415 \beta_{m} K_{2}}\right) \text {, } \\
& 3 N_{b}\left(E_{c}+K_{1}\right. \\
& \left.\cdot \frac{d_{4}^{\alpha}\left[\ln \left(1-C^{1 / n N_{b}}\right)-\ln \left(0.1826 \alpha_{m}\right)\right]}{-0.5415 \beta_{m} K_{2}}\right) \\
& =2 N_{b}\left(E_{c}+K_{1}\right. \\
& \left.\cdot \frac{d_{4}^{\alpha}\left[\ln \left(1-C^{1 / n N_{b}}\right)-\ln \left(0.1826 \alpha_{m}\right)\right]}{-0.5415 \beta_{m} K_{2}}\right),
\end{aligned}
$$

$$
\begin{aligned}
2 N_{b}\left(E_{c}+K_{1}\right. \\
. \\
\left.\quad \frac{d_{4}^{\alpha}\left[\ln \left(1-C^{1 / n N_{b}}\right)-\ln \left(0.1826 \alpha_{m}\right)\right]}{-0.5415 \beta_{m} K_{2}}\right) \\
=N_{b}\left(E_{c}+K_{1}\right. \\
\left.\quad \cdot \frac{d_{5}^{\alpha}\left[\ln \left(1-C^{1 / n N_{b}}\right)-\ln \left(0.1826 \alpha_{m}\right)\right]}{-0.5415 \beta_{m} K_{2}}\right), \\
d_{1}+d_{2}+d_{3}+d_{4}+d_{5}+d_{6}=D .
\end{aligned}
$$

Substituting all known parameters into the above equations we can get

$$
\begin{aligned}
& d_{1}=49, \\
& d_{2}=60, \\
& d_{3}=71, \\
& d_{4}=84, \\
& d_{5}=102, \\
& d_{6}=135 .
\end{aligned}
$$

Then the length of each hop is obtained, and the energy consumption for each node is balanced; that is,

$$
\begin{aligned}
E_{1}^{\prime} & =N_{b} n\left(E_{c}\right. \\
& \left.+\frac{K_{1} d_{1}^{\alpha}\left[\ln \left(1-C^{1 / n N_{b}}\right)-\ln \left(0.1826 \alpha_{m}\right)\right]}{-0.5415 \beta_{m} K_{2}}\right) .
\end{aligned}
$$

If nodes are equidistantly deployed, $d_{1}=D / n=83.33$.

If nodes are not equidistantly deployed, $d_{1}=49$.

Comparing these two schemes, the proportion of declined energy is

$$
\phi_{1}=\frac{E_{1}^{\mathrm{AWGN}}-E_{1}^{\mathrm{AWGN}{ }^{\prime}}}{E_{1}^{\mathrm{AWGN}}}=50 \% .
$$

On the other hand, the energy consumption for node $i$ is also associated with the reliability $c_{i}$; namely, the higher reliability leads to more energy consumption, and we can decrease energy consumption by decreasing node reliability. Since the reliability of the entire routing must be ensured as $C$, if the reliability of the node with maximum energy consumption is decreased, we must increase reliability for nodes with less energy consumption correspondingly; then, we get Theorem 7.

Theorem 7. For multisource linear network under AWGN channels, to solve a set of $c_{1} \leq c_{2} \leq c_{3} \leq \cdots \leq c_{n}$ which achieves $\min \max \left(E_{i, 2}\right) \mid i \in\{1, \ldots, n\}$. 
S.t. $\prod_{i=1}^{n} C_{i}=C, d_{1}=d_{2}=\cdots=d_{n}, d_{1}+d_{2}+\cdots+d_{n}=D$. It can be converted into the following:

$$
\begin{aligned}
& T+n M_{2} \ln \left(1-C_{1}^{N}\right)-(n-1) M_{2} \ln \left(1-C_{2}^{N}\right)=0, \\
& T+(n-1) M_{2} \ln \left(1-C_{2}^{N}\right) \\
& \quad-(n-2) M_{2} \ln \left(1-C_{3}^{N}\right)=0, \\
& \vdots \\
& T+2 M_{2} \ln \left(1-C_{n-1}^{N}\right)-M_{2} \ln \left(1-C_{n}^{N}\right)=0, \\
& \prod_{i=1}^{n} C_{i}=C, \\
& M_{2}=\frac{K_{1} d_{h o p}^{\alpha}}{-0.5415 \beta_{m} K_{2}}, \\
& T=E_{c}-M_{2} \ln \left(0.1826 \alpha_{m}\right) .
\end{aligned}
$$

Proof. According to the above conditions, it yields

$$
E_{i, 2}=N_{b}(n+1-i)\left(E_{c}+K_{1} \cdot P_{t_{i}, 2}\right),
$$

where

$$
P_{t, 2}=\frac{d_{\text {hop }}^{\alpha}\left[\ln \left(1-C_{i}^{1 / n N_{b}}\right)-\ln \left(0.1826 \alpha_{m}\right)\right]}{-0.5415 \beta_{m} K_{2}}
$$

and $\prod_{i=1}^{n} C_{i}=C$.

Assume there are $n$ nodes; if the energy consumption is balanced, we get the following equation set:

$$
\begin{gathered}
E_{1}=E_{2}, \\
E_{2}=E_{3}, \\
\vdots \\
E_{n-1}=E_{n}, \\
\prod_{i=1}^{n} C_{i}=C .
\end{gathered}
$$

Substitute (73) into (72); that is,

$$
\begin{aligned}
E_{i, 2} & =N_{b}(n+1-i)\left(E_{c}+K_{1}\right. \\
& \left.\cdot \frac{d_{\text {hop }}^{\alpha}\left[\ln \left(1-C_{i}^{1 / n N_{b}}\right)-\ln \left(0.1826 \alpha_{m}\right)\right]}{-0.5415 \beta_{m} K_{2}}\right) .
\end{aligned}
$$

Set $M_{2}=K_{1} d_{\text {hop }}^{\alpha} /-0.5415 \beta_{m} K_{2}$.

Substitute the above equation into (75); that is,

$$
\begin{aligned}
& E_{i, 2}=N_{b}(n+1-i) \\
& \quad \cdot\left(E_{c}+M_{2}\left[\ln \left(1-C_{i}^{1 / n N_{b}}\right)-\ln \left(0.1826 \alpha_{m}\right)\right]\right) .
\end{aligned}
$$

Reorganizing the above formula, we can get

$$
\begin{aligned}
& E_{i, 2}=N_{b}(n+1-i) \\
& \quad \cdot\left(E_{c}+M_{2} \ln \left(1-C_{i}^{1 / n N_{b}}\right)-M_{2} \ln \left(0.1826 \alpha_{m}\right)\right) .
\end{aligned}
$$

Set $T=E_{c}-M_{2} \ln \left(0.1826 \alpha_{m}\right)$.

Substitute the above equation into (77); that is,

$$
E_{i, 2}=N_{b}(n+1-i)\left(T+M_{2} \ln \left(1-C_{i}^{1 / n N_{b}}\right)\right) .
$$

Set $n_{1}=1 / n N_{b}$.

Substitute the above equation into (78); that is,

$$
E_{i, 2}=N_{b}(n+1-i)\left(T+M_{2} \ln \left(1-C_{i}^{n_{1}}\right)\right) .
$$

Substitute (79) into (74); that is,

$$
\begin{aligned}
& n\left(T+M_{2} \ln \left(1-C_{1}^{n_{1}}\right)\right) \\
& \quad=(n-1)\left(T+M_{2} \ln \left(1-C_{2}^{n_{1}}\right)\right), \\
& (n-1)\left(T+M_{2} \ln \left(1-C_{2}^{n_{1}}\right)\right) \\
& \quad=(n-2)\left(T+M_{2} \ln \left(1-C_{3}^{n_{1}}\right)\right),
\end{aligned}
$$

$$
\begin{aligned}
& 2\left(T+M_{2} \ln \left(1-C_{n-1}^{n_{1}}\right)\right)=\left(T+M_{2} \ln \left(1-C_{n}^{n_{1}}\right)\right), \\
& \prod_{i=1}^{n} C_{i}=C .
\end{aligned}
$$

Reorganizing the above equations yields

$$
\begin{aligned}
& T+n M_{2} \ln \left(1-C_{1}^{n_{1}}\right)-(n-1) M_{2} \ln \left(1-C_{2}^{n_{1}}\right)=0, \\
& T+(n-1) M_{2} \ln \left(1-C_{2}^{n_{1}}\right) \\
& \quad-(n-2) M_{2} \ln \left(1-C_{3}^{n_{1}}\right)=0, \\
& \vdots \\
& T+2 M_{2} \ln \left(1-C_{n-1}^{n_{1}}\right)-M_{2} \ln \left(1-C_{n}^{n_{1}}\right)=0, \\
& \prod_{i=1}^{n} C_{i}=C .
\end{aligned}
$$

Inference 2. The solvability criterion of (71) is as follows: if $T / M_{2}<0$, then (71) is solvable, where

$$
\begin{aligned}
T & =E_{c}-M_{2} \ln \left(0.1826 \alpha_{m}\right), \\
M_{2} & =\frac{K_{1} d_{\text {hop }}^{\alpha}}{-0.5415 \beta_{m} K_{2}} .
\end{aligned}
$$

Proof. Computing the front $n-1$ equations of (71) yields

$$
C_{i+1}^{n_{1}}=1-\left(1-C_{1}^{n_{1}}\right)^{n /(n-i)} \exp \left(\frac{i T}{(n-i) M_{2}}\right)
$$


Substituting the above equation into the last equation of (71), we can get

$$
\begin{aligned}
C_{1}^{n_{1}} & \cdot \prod_{i=1}^{n-1}\left(1-\left(1-C_{1}^{n_{1}}\right)^{n /(n-i)} \exp \left(\frac{i T}{(n-i) M_{2}}\right)\right) \\
& =C^{n_{1}} .
\end{aligned}
$$

Set

$$
\begin{aligned}
& H_{1}\left(C_{1}\right) \\
& =C_{1}^{n_{1}} \prod_{i=1}^{n-1}\left(1-\left(1-C_{1}^{n_{1}}\right)^{n /(n-i)} \exp \left(\frac{i T}{(n-i) M_{2}}\right)\right) \\
& \quad-C^{n_{1}} .
\end{aligned}
$$

Obviously, $H_{1}\left(C_{1}\right)$ is a monotone increasing function in $C_{1} \in[0,1]$ (see proof below), and when $C_{1}=0, H_{1}\left(C_{1}\right)<0$; when $C_{1}=1, H_{1}\left(C_{1}\right)>0$. Since $H_{1}\left(C_{1}\right)$ is a continuous function, then there must exist a solution in $C_{1} \in[0,1]$ to make $H_{1}\left(C_{1}\right)=0$. The following proves (88) to be an increasing function. First, through analysis, we can know that $E_{i}\left(C_{1}\right)=1-\left(1-C_{1}^{n_{1}}\right)^{n /(n+1-i)} \exp \left(i T /(n-i) M_{2}\right)(i \geq 2)$ is a monotone increasing function in $C_{1} \in[0,1]$; if $T / M_{2}<0$, then $0<\exp \left(i T /(n-i) M_{2}\right)<1$, while $0<\left(1-C_{1}^{n_{1}}\right)^{n /(n+1-i)}<$ 1 ; thus $0<E_{i}\left(C_{1}\right)$; therefore, (85) is an increasing function, and it is solvable.

For instance, assuming $D=500, n=6$, and $C=0.9$, we can know that $T / M_{2}<0$; that is, the origin equation set is solvable, and (79) can be converted into the following:

$$
\begin{gathered}
T+6 M_{2} \ln \left(1-C_{1}^{n_{1}}\right)-5 M_{2} \ln \left(1-C_{2}^{n_{1}}\right)=0, \\
T+5 M_{2} \ln \left(1-C_{2}^{n_{1}}\right)-4 M_{2} \ln \left(1-C_{3}^{n_{1}}\right)=0, \\
\vdots \\
T+2 M_{2} \ln \left(1-C_{5}^{n_{1}}\right)-M_{2} \ln \left(1-C_{6}^{n_{1}}\right)=0, \\
\prod_{i=1}^{6} C_{i}=C .
\end{gathered}
$$

Correspondingly, it yields

$$
\begin{aligned}
& C_{i+1}^{n_{1}}=1-\left(1-C_{1}^{n_{1}}\right)^{6 /(6-i)} \exp \left(\frac{i T}{(6-i) M_{2}}\right), \\
& C_{1}^{n_{1}} \cdot \prod_{i=1}^{5}\left(1-\left(1-C_{1}^{n_{1}}\right)^{6 /(6-i)} \exp \left(\frac{i T}{(6-i) M_{2}}\right)\right) \\
& =C^{n_{1}} .
\end{aligned}
$$

Substituting the parameters into the above yields $C_{1}=$ $0.904, C_{2}=0.9962$, and $C_{3}=\cdots=C_{6}=1$.

4.3. Grid Network. In this section, we extend the linear network to a two-dimensional network, which is a mesh network [24]. In this kind of network, nodes are regularly deployed

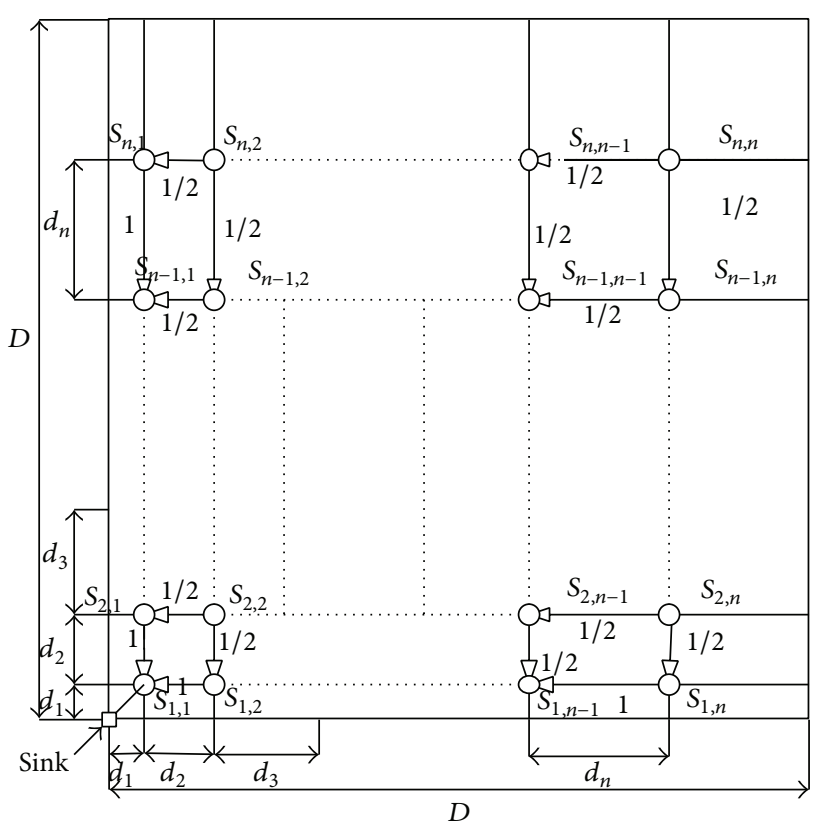

FIGURE 7: Grid network.

in intersections of rows and columns; the sink is located in the intersection of bottom left row and column, as shown in Figure 7. In a grid network, each node generates one data packet transmitting to the sink, and the transmission direction is restricted in downward or leftward direction with the same probability. This section first calculates the energy consumption for each node in the network and then discusses how to process cross layer optimization for grid network.

Theorem 8. In grid network, the node's data load is

$$
\begin{aligned}
& B_{n, n}=1, \\
& B_{n, j}=2-\left(\frac{1}{2}\right)^{n-j} \quad(1 \leq j \leq n-1), \\
& B_{i, 1}=1+B_{i+1,1}+\frac{1}{2} B_{i, 2} \quad(1 \leq i \leq n-1), \\
& B_{i, j}=1+\frac{1}{2}\left(B_{i+1, j}+B_{i, j+1}\right) \quad(1<i, j<n), \\
& B_{i, j}=B_{j, i} \quad(i \neq j) .
\end{aligned}
$$

Proof. The size of $B_{i, j}$ denotes the data amount received by $S_{i, j}$. First, we analyze $S_{n, j}$. Since $B_{n, n}=1$, then $B_{n, n-1}=$ $1+1 / 2=3 / 2$, and $B_{n, n-2}=1+(1 / 2) B_{n, n-1}=7 / 4$; we get $B_{n, j}=1+(1 / 2) B_{n, j+1}=2-(1 / 2)^{n-j}$, in which the $n$th row is determined. Then we analyze the $(n-1)$ th row and get $B_{n-1, j}=1+(1 / 2)\left(B_{n, j}+B_{n-1, j+1}\right)$ when $(2 \leq j \leq n-1)$. While $B_{n-1,1}=1+B_{n, 1}+(1 / 2) B_{n-1,2}$, the $(n-1)$ th row is determined. Therefore, Theorem 8 is proved.

Different from what was discussed in the linear networks, the grid network is composed of multirows (columns) of nodes, and the data load for each node is not the same; then 
the optimization is more complex. Since the network lifetime is determined by the lifetime of node which has the maximum energy consumption, and the grid network is symmetrical, therefore if the performance of a row or column which has the maximum energy consumption is optimized, then the lifetime of the entire network is maximized. Theorem 8 proves that, in the grid network, nodes in the first row (column) have the heaviest data load.

Theorem 9. In grid network, each node in the first row (column) has bigger data load than other nodes in the same row.

Proof. According to the second equation in (88), we can get the data load for each node in the $n$th row; obviously, $B_{n, i}>$ $B_{n, j}(i<j)$; then, we analyze the $(n-1)$ th row, according to the symmetry of the second formula in (88), yielding

$$
\begin{aligned}
B_{n-1, n-1} & =1+\frac{1}{2}\left(B_{n, n-1}+B_{n-1, n}\right)=1+B_{n, n-1}, \\
B_{n-1, n-2} & =1+\frac{1}{2}\left(B_{n, n-2}+B_{n-1, n-1}\right), \\
B_{n, n-1} & <B_{n, n-2}, \\
B_{n, n-1} & <B_{n-1, n-1}, \\
B_{n-1, n-2} & >B_{n-1, n-1} .
\end{aligned}
$$

Similarly, we get $B_{n-1,1}>B_{n-1,2}$ and $B_{i, 1}>B_{i, 2}(1 \leq i \leq n)$ to prove Theorem 9.

Inference 3. In grid network, each node in the first row (column) has higher energy consumption than other nodes in the same row.

Proof. By now, we can get the data load of each node, and we know that the first row and the first column have the biggest data load; then we analyze the first column. If we get the data load for each node in the first column, according to the condition of balanced energy consumption, we get $n$ equations and we can obtain corresponding $d_{i}(1 \leq i \leq n)$, and the node energy consumption is the same for the first column. Then we can substitute $d_{i}(1 \leq i \leq n)$ into the second column. Due to the symmetry, we can only consider the energy consumption of $S_{n, 2}, \ldots, S_{2,2}, S_{2,1}$ when analyzing the second column. As can be known from the above proof, we get $B_{i, 1}>B_{i, 2}$; according to the formula, we get $E_{i, j} \propto d_{i}^{3}$ and $E_{i, j} \propto B_{i, j}$; then we get $E_{i, 1}>E_{i, 2}$. Then the node with the highest energy consumption in the second column is $S_{2,1}$. Similarly, the node with highest energy consumption in the third column is $S_{3,1}$ and so on and the node with highest energy consumption in the $n$th column is $S_{n, 1}$, which shows that, for $d_{i}(1 \leq i \leq n)$, other nodes have less energy consumption except for the first column.

This section discusses optimization in AWGN channels for grid networks. In such networks, the number of nodes is determined, that is, $n \times n$, and thus the deployment cost is determined; the optimization goal is how to maximize the network lifetime. Factors that can be optimized are the placement of nodes $d^{*}$ and node transmission structure $P^{*}$. In this paper, we propose two optimization methods: one is to optimize $d^{*}$ and the other is to optimize $P^{*}$; through these two methods, we can maximize network lifetime. In Theorem 10, we give the conclusion of node placement optimization.

Theorem 10. In AWGN grid network, the energy consumption of nodes in maximum consumption row (column) can be balanced if $d_{i}$ of node i meets the following:

$$
\begin{gathered}
\left(B_{1,1}-B_{2,1}\right) E_{c}+B_{1,1} M_{3}\left(\sqrt{2} d_{1}\right)^{\alpha}-B_{2,1} M_{3} d_{2}^{\alpha}=0, \\
\left(B_{2,1}-B_{3,1}\right) E_{c}+B_{2,1} M_{3} d_{2}^{\alpha}-B_{3,1} M_{3} d_{3}^{\alpha}=0, \\
\vdots \\
\left(B_{n-1,1}-B_{n, 1}\right) E_{c}+B_{n-1,1} M_{3} d_{n-1}^{\alpha}-B_{n, 1} M_{3} d_{n}^{\alpha}=0, \\
\sum_{i=1}^{n} d_{i}=D .
\end{gathered}
$$

Proof. We can calculate the data amount of each node according to (90). Therefore, we can get the optimal $d_{i}(1 \leq$ $i \leq n)$ if the first column is optimized.

First, solve optimal $d_{i}(1 \leq i \leq n), E_{i, 1}=N_{b}\left(E_{c}+\right.$ $\left.K_{1} \cdot P_{t_{i}}\right) B_{i, 1}$, which is the same as previous analysis in linear network; it is optimal when the energy consumption is balanced and then yields

$$
\begin{gathered}
E_{1,1}=E_{2,1}, \\
E_{2,1}=E_{3,1}, \\
\vdots \\
E_{n-1,1}=E_{n, 1}, \\
\sum_{i=1}^{n} d_{i}=D .
\end{gathered}
$$

As can be known from previous analysis, it yields

$$
P_{t_{i}}^{\mathrm{AWGN}}=\frac{d_{i}^{\alpha}\left[\ln \left(1-C^{1 /(2 n-1) N_{b}}\right)-\ln \left(0.1826 \alpha_{m}\right)\right]}{-0.5415 \beta_{m} K_{2}} .
$$

Then

$$
\begin{aligned}
& E_{t, 1}^{\mathrm{AWGN}}=N_{b} B_{i, 1}\left(E_{c}\right. \\
& \left.\quad+\frac{K_{1} d_{i}^{\alpha}\left[\ln \left(1-C^{1 /(2 n-1) N_{b}}\right)-\ln \left(0.1826 \alpha_{m}\right)\right]}{-0.5415 \beta_{m} K_{2}}\right) .
\end{aligned}
$$



then

Since transmission distance from $S_{1,1}$ to sink is $\sqrt{2} d_{1}$,

$$
\begin{aligned}
& E_{1,1}^{\mathrm{AWGN}}=N_{b} B_{1,1}\left(E_{c}\right. \\
& \left.+\frac{K_{1}\left(\sqrt{2} d_{1}\right)^{\alpha}\left[\ln \left(1-C^{1 /(2 n-1) N_{b}}\right)-\ln \left(0.1826 \alpha_{m}\right)\right]}{-0.5415 \beta_{m} K_{2}}\right) .
\end{aligned}
$$

Set

$$
M_{3}=K_{1} \cdot \frac{\left[\ln \left(1-C^{1 /(2 n-1) N_{b}}\right)-\ln \left(0.1826 \alpha_{m}\right)\right]}{-0.5415 \beta_{m} K_{2}} .
$$

Substituting the above formula into (93), we get

$$
\begin{aligned}
& E_{i, 1}=N_{b} B_{i, 1}\left(E_{c}+M_{3} d_{i}^{\alpha}\right), \\
& E_{1,1}=N_{b} B_{1,1}\left(E_{c}+M_{3}\left(\sqrt{2} d_{1}\right)^{\alpha}\right) .
\end{aligned}
$$

Substituting the above formula into the equation set, we get (90).

Inference 4. The solvability criterion of (93) is as follows.

If $g_{3}(\varepsilon)=\varepsilon+\sum_{i=2}^{n}\left(\left(B_{1,1} M_{3}(\sqrt{2} \varepsilon)^{\alpha}+\left(B_{1,1}-B_{i, 1}\right) E_{c}\right) /\right.$ $\left.M_{3} B_{i, 1}\right)^{1 / \alpha}-D \leq 0 \mid \varepsilon \rightarrow 0$ then (93) is solvable.

Proof. Computing the front $n-1$ equations of (93), we can get

$$
d_{i}=\left(\frac{B_{1,1} M_{3}\left(\sqrt{2} d_{1}\right)^{\alpha}+\left(B_{1,1}-B_{i, 1}\right) E_{c}}{M_{3} B_{i, 1}}\right)^{1 / \alpha}
$$

$$
(i \geq 1) \text {. }
$$

Represent all $d_{i}$ with $d_{1}\left(D \geq d_{1}>0\right)$ and then substitute them into the $n$th equation in (93); that is,

$$
\begin{aligned}
d_{1} & +\sum_{i=2}^{n}\left(\frac{B_{1,1} M_{3}\left(\sqrt{2} d_{1}\right)^{\alpha}+\left(B_{1,1}-B_{i, 1}\right) E_{c}}{M_{3} B_{i, 1}}\right)^{1 / \alpha} \\
& =D .
\end{aligned}
$$

Set

$$
g_{3}\left(d_{1}\right)
$$$$
=d_{1}
$$$$
+\sum_{i=2}^{n}\left(\frac{B_{1,1} M_{3}\left(\sqrt{2} d_{1}\right)^{\alpha}+\left(B_{1,1}-B_{i, 1}\right) E_{c}}{M_{3} B_{i, 1}}\right)^{1 / \alpha}
$$

Through analysis, we can know that $g_{3}\left(d_{1}\right)$ is a monotone increasing function in $d_{1} \in(0, D]$. Obviously, when $d_{1}=D$, we can get $g_{3}\left(d_{1}\right)>0$, and when $d_{1}=\varepsilon \mid \varepsilon \rightarrow 0$, it yields

$$
\begin{aligned}
& g_{3}(\varepsilon) \\
& \quad=\varepsilon+\sum_{i=2}^{n}\left(\frac{B_{1,1} M_{3}(\sqrt{2} \varepsilon)^{\alpha}+\left(B_{1,1}-B_{i, 1}\right) E_{c}}{M_{3} B_{i, 1}}\right)^{1 / \alpha}
\end{aligned}
$$

$-D$.

Therefore, only if (100) is not bigger than 0 does the original equation have solutions and we can get the appropriate solution.

To further reduce the energy consumption near the sink, based on the first stage, we reduce the node reliability near the sink and increase the node reliability far from the sink to achieve total reliability $\prod_{i=1}^{k} \gamma_{i} \geq C$.

Theorem 11. In AWGN grid network, the energy consumption of nodes in maximum consumption row (column) can be balanced if reliability $c_{i}$ of node $i$ meets the following:

$$
\begin{aligned}
& \left(B_{1,1}-B_{2,1}\right) E_{c} \\
& +B_{1,1} M_{4}\left(\sqrt{2} d_{1}\right)^{\alpha}\left[\ln \left(1-C_{1}^{1 /(2 n-1) N_{b}}\right)\right] \\
& -B_{2,1} M_{4} d_{2}^{\alpha}\left[\ln \left(1-C_{2}^{1 /(2 n-1) N_{b}}\right)\right]=0, \\
& \left(B_{2,1}-B_{3,1}\right) E_{c}+B_{2,1} M_{4} d_{2}^{\alpha}\left[\ln \left(1-C_{2}^{\left.1 /(2 n-1) N_{b}\right)}\right]\right. \\
& -B_{3,1} M_{4} d_{3}^{\alpha}\left[\ln \left(1-C_{3}^{1 /(2 n-1) N_{b}}\right)\right]=0, \\
& \quad \vdots \\
& \left(B_{n-1,1}-B_{n, 1}\right) E_{c} \\
& +B_{n-1,1} M_{4} d_{n-1}^{\alpha}\left[\ln \left(1-C_{n-1}^{1 /(2 n-1) N_{b}}\right)\right] \\
& \quad-B_{n, 1} M_{4} d_{n}^{\alpha}\left[\ln \left(1-C_{n}^{1 /(2 n-1) N_{b}}\right)\right]=0, \\
& \prod_{i=1}^{n} C_{i} \geq C .
\end{aligned}
$$

Proof. As can be known from previous analysis, if $d_{i}(1 \leq i \leq$ $n$ ) is obtained, then the problem

$$
P_{t_{i}}^{\prime}=\frac{d_{i}^{\alpha}\left[\ln \left(1-C_{i}^{1 /(2 N-1) N_{b}}\right)-\ln \left(0.1826 \alpha_{m}\right)\right]}{-0.5415 \beta_{m} K_{2}}
$$

is converted to solve optimal $C_{i}(1 \leq i \leq n)$, which is the same as previous analysis on linear network; it is optimal when the energy consumption is balanced; then it yields

$$
E_{1,1}=E_{2,1} \text {, }
$$




$$
\begin{gathered}
E_{2,1}=E_{3,1}, \\
\vdots \\
E_{n-1,1}=E_{n, 1}, \\
\prod_{i=1}^{n} C_{i} \geq C .
\end{gathered}
$$

Then, it yields

$$
\begin{aligned}
E_{i, 1} & =N_{b} B_{i, 1}\left(E_{c}\right. \\
& \left.+\frac{K_{1} d_{i}^{\alpha}\left[\ln \left(1-C_{i}^{1 /(2 n-1) N_{b}}\right)-\ln \left(0.1826 \alpha_{m}\right)\right]}{-0.5415 \beta_{m} K_{2}}\right),
\end{aligned}
$$

where $i \neq 1$.
Set

$$
M_{4}=\frac{K_{1}}{-0.5415 \beta_{m} K_{2}} \text {. }
$$

Substituting the above formula into (104) yields

$$
\begin{aligned}
E_{i, 1} & =N_{b} B_{i, 1}\left(E_{c}\right. \\
+ & \left.M_{4} d_{i}^{\alpha}\left[\ln \left(1-C_{i}^{1 /(2 n-1) N_{b}}\right)-\ln \left(0.1826 \alpha_{m}\right)\right]\right) \\
E_{1,1} & =N_{b} B_{1,1}\left(E_{c}+M_{4}\left(\sqrt{2} d_{1}\right)^{\alpha}\right. \\
\cdot & {\left.\left[\ln \left(1-C_{1}^{1 /(2 n-1) N_{b}}\right)-\ln \left(0.1826 \alpha_{m}\right)\right]\right) . }
\end{aligned}
$$
(103).

Substituting the above formula into the equation set yields

First, computing the front $n-1$ equations of the above equation set yields

$$
C_{i}^{1 /(2 n-1) N_{b}}=1-\exp \left(\frac{\left(B_{1,1}-B_{i, 1}\right) E_{c}+M_{4} B_{1,1}\left(\sqrt{2} d_{1}\right)^{\alpha}\left[\ln \left(1-C_{1}^{1 /(2 n-1) N_{b}}\right)+1.7\right]}{M_{4} B_{i, 1} d_{i}^{\alpha}}-1.7\right) \quad(i \geq 2) .
$$

Represent all $C_{i}$ with $C_{1}$, and then substitute them into the $n$th equation; that is,

$$
C_{1}^{1 /(2 n-1) N_{b}} \prod_{i=2}^{n}\left\{1-\exp \left(\frac{\left(B_{1,1}-B_{i, 1}\right) E_{c}+M_{4} B_{1,1}\left(\sqrt{2} d_{1}\right)^{\alpha}\left[\ln \left(1-C_{1}^{1 /(2 n-1) N_{b}}\right)+1.7\right]}{M_{4} B_{i, 1} d_{i}^{\alpha}}-1.7\right)\right\}=C^{1 /(2 n-1) N_{b}} .
$$

$$
\begin{aligned}
H_{4}\left(C_{1}\right)= & C_{1}^{1 /(2 n-1) N_{b}} \prod_{i=2}^{n}\left\{1-\exp \left(\frac{\left(B_{1,1}-B_{i, 1}\right) E_{c}+M_{4} B_{1,1}\left(\sqrt{2} d_{1}\right)^{\alpha}\left[\ln \left(1-C_{1}^{1 /(2 n-1) N_{b}}\right)+1.7\right]}{M_{4} B_{i, 1} d_{i}^{\alpha}}-1.7\right)\right\} \\
& -C^{1 /(2 n-1) N_{b}} .
\end{aligned}
$$

Obviously, when $C_{1}=0$, we get $H_{4}\left(C_{1}\right)<0$, and when $C_{1}=$ 1 , we get $H_{4}\left(C_{1}\right)>0$; since $H_{4}\left(C_{1}\right)$ is a continuous function, then there must exist a $C_{1} \in[0,1]$ which achieves $H_{4}\left(C_{1}\right)=0$.

\section{Performance Analysis and Experimental Results}

This section provides some simulation examples to verify the adaptive sensing range optimal design proposed in this paper.

Figure 8 shows the energy consumption under AWGN channels, which includes energy consumed by transmitting circuit and transmit amplifier energy consumption; as can be seen, as the transmission distance increases, the transmit amplifier energy consumption increases rapidly but the transmitting circuit declines; thus there exists an optimal design of transmission distance to minimize node transmitting energy consumption.

5.1. Multisource Linear Network. We define minimum total energy consumption for transmitting unit bit data to the sink as MTEC and define minimum per node energy consumption for transmitting unit bit data to the sink as MPNEC. 


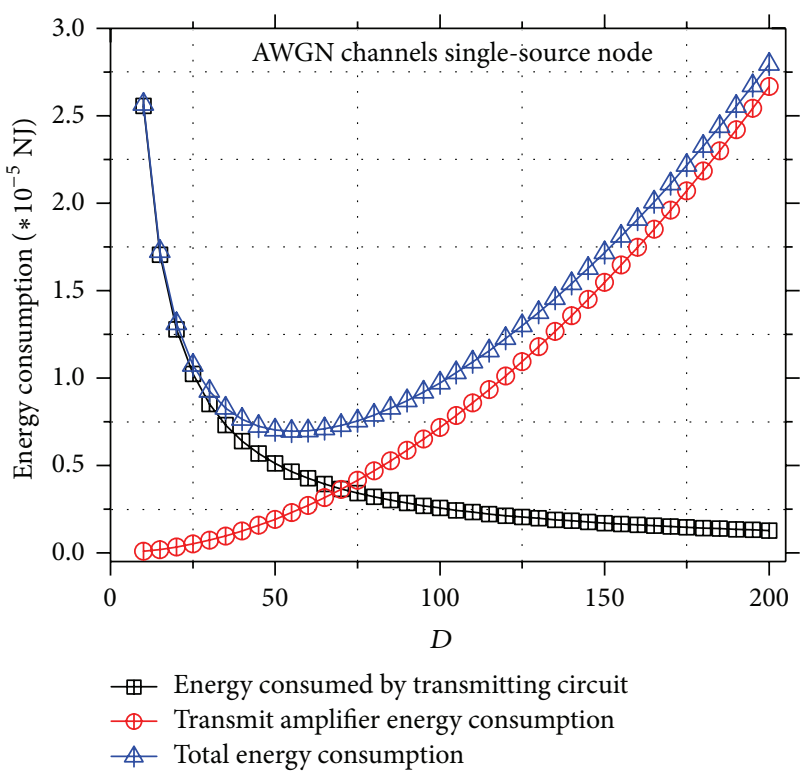

FIGURE 8: Node energy consumption under AWGN channels.

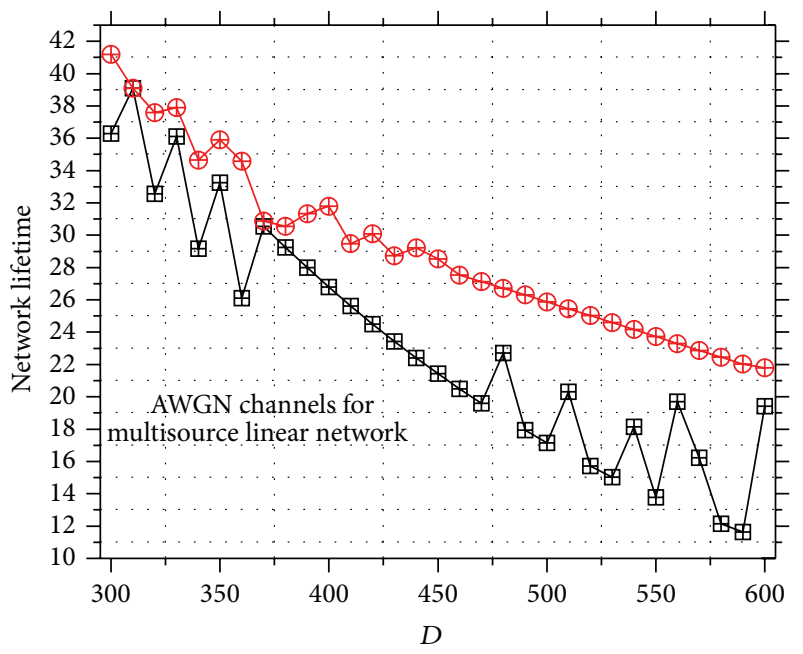

\#- The policy for min total energy consumption

-1) The policy for min per node energy consumption

FIgUre 9: The energy consumption of MTEC versus MPNEC.

Figure 9 shows the comparison of network lifetime under different optimization methods. As can be seen from Figure 9, the network lifetime in MPNEC scheme is higher than that in the MTEC scheme. In Figure 10, we can see that MPNEC scheme can improve the network lifetime by $30 \%$ averagely compared with the MTEC scheme. That is because in MTEC the goal is to minimize total energy consumption, and it does not care about how many nodes are needed; then the cost for per unit data can be higher, and the utilization is lower. While MPNEC aims at optimizing the total transmitting energy divided by node number, then its utilization is optimal, and thus it can achieve higher network lifetime than MTEC. Figure 11 shows the optimal node number to obtain optimization in different schemes when $D$ is determined. As can be

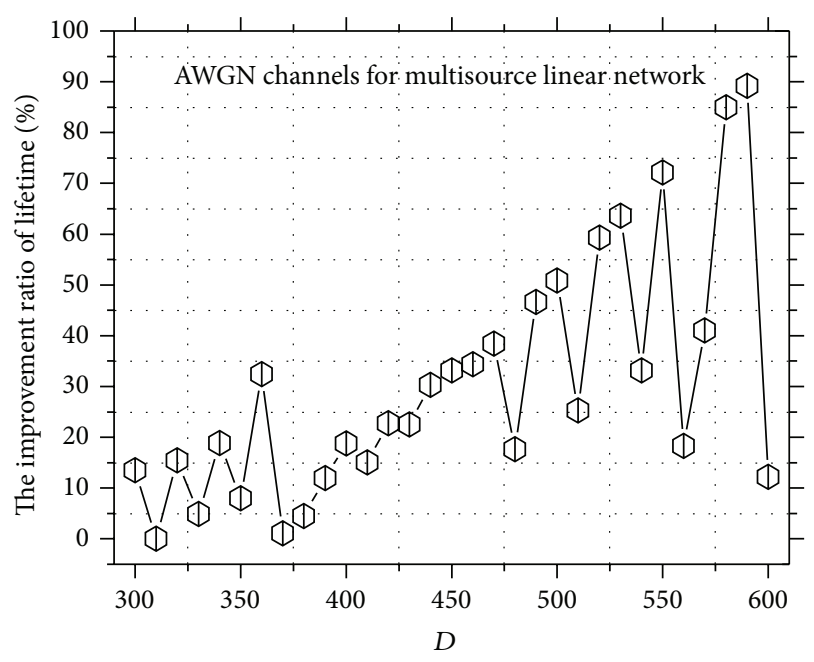

FIgURE 10: The lifetime improvement ratio of MPNEC versus MTEC.

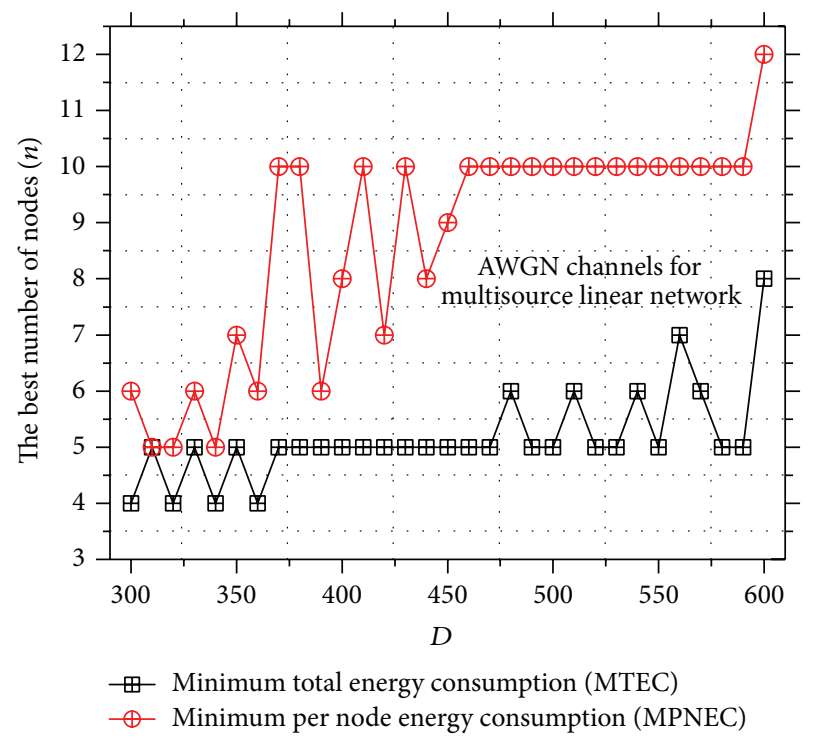

FIGURE 11: The optimal node number under MPNEC and MTEC.

seen, the optimal node number is different under different schemes.

In this paper, we propose an adaptive sensing range optimal policy to achieve balanced network energy consumption, which adopts shorter transmission distance for nodes near the sink and adopts farther transmission distance for nodes far from the sink; we call it ASTDN (adaptive sensing transmission distance of nodes), while, in previous research where nodes have equal transmission distance, it is called USTDN (unadaptive sensing transmission distance of nodes). In ASTDN, after the optimal node number is obtained, we deploy nodes with unequal transmission distance to balance energy consumption and thus improve network lifetime. Table 2 shows the unequal transmission distance of nodes in ASTDN. 
TABLE 2: The unequal transmission distance of nodes (AWGN channels).

\begin{tabular}{cccccccccc}
\hline$d_{1}$ & $d_{2}$ & $d_{3}$ & $d_{4}$ & $d_{5}$ & $d_{6}$ & $d_{7}$ & $d_{8}$ & $d_{9}$ & $D$ \\
\hline 40 & 43 & 45 & 47 & 50 & 54 & 59 & 68 & 85 & 491 \\
41 & 44 & 46 & 48 & 51 & 55 & 61 & 69 & 87 & 502 \\
42 & 45 & 47 & 50 & 53 & 57 & 62 & 71 & 89 & 516 \\
43 & 46 & 48 & 51 & 54 & 58 & 64 & 72 & 91 & 527 \\
44 & 47 & 49 & 52 & 55 & 59 & 65 & 74 & 93 & 538 \\
45 & 48 & 50 & 53 & 56 & 60 & 66 & 76 & 95 & 549 \\
46 & 49 & 52 & 54 & 57 & 62 & 68 & 77 & 97 & 562 \\
47 & 50 & 53 & 55 & 59 & 63 & 69 & 79 & 99 & 574 \\
48 & 51 & 54 & 56 & 60 & 64 & 71 & 81 & 101 & 586 \\
49 & 52 & 55 & 58 & 61 & 66 & 72 & 82 & 103 & 598 \\
50 & 54 & 56 & 59 & 62 & 67 & 74 & 84 & 106 & 612 \\
\hline
\end{tabular}

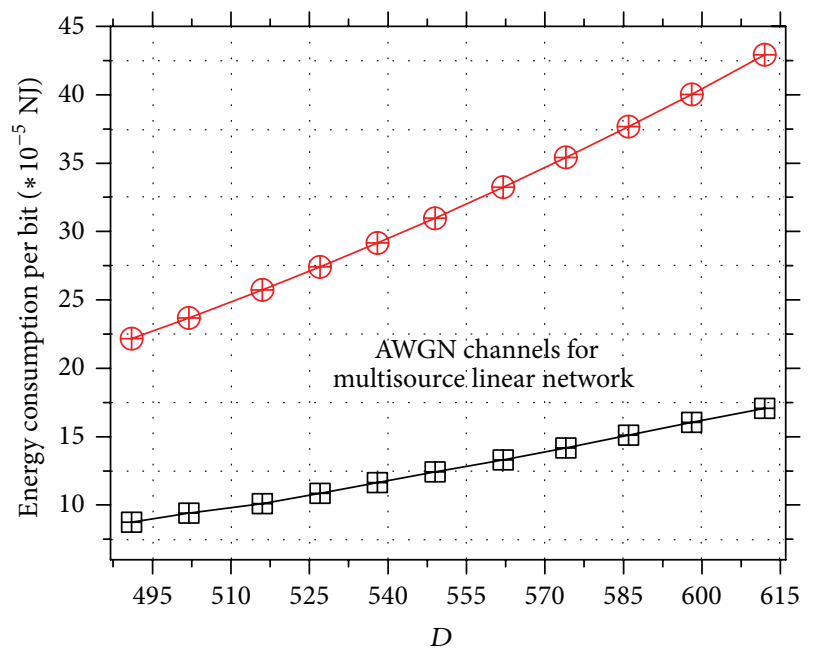

巴 Adaptive sensing transmission distance of nodes (ASTDN)

(1) Unadaptive sensing transmission distance of nodes (USTDN)

FIGURE 12: The energy consumption under ASTDN and USTDN.

Figure 12 shows the energy consumption under ASTDN and USTDN, combined with Figure 13; we can know that the energy consumption with our ASTDN is less than half of USTDN.

In addition, the adaptive sensing range optimal approach proposed in this paper adopts lower reliability requirement for nodes near the sink and higher reliability requirement for nodes far from the sink under the premise that total reliability meets the requirement of applications, which can improve network lifetime as well. We call it ASRN (adaptive sensing reliability of nodes), while, in previous research where nodes have equal reliability, it is called USRN (unadaptive sensing reliability of nodes). Table 3 shows the nodes reliability; as can be seen, under ASRN, the reliability of nodes near the sink is lower and that of nodes far from the sink is higher, and the network total reliability is 0.828 , while, under USRN, the reliability of each node should be 0.977 to achieve network total reliability as 0.828 . The node with highest energy consumption has higher reliability under USRN over ASRN, and thus

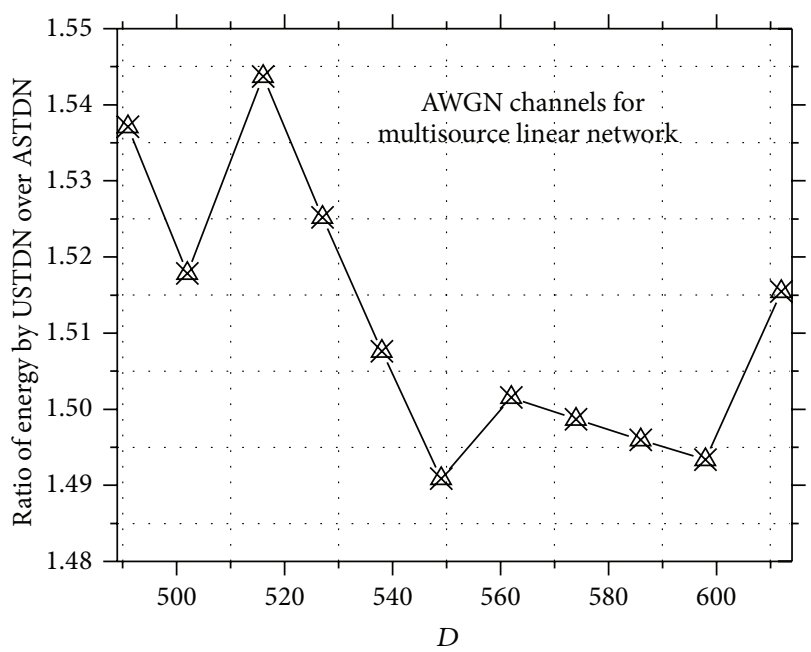

FIGURE 13: The ratio of energy by USTDN over ASTDN.

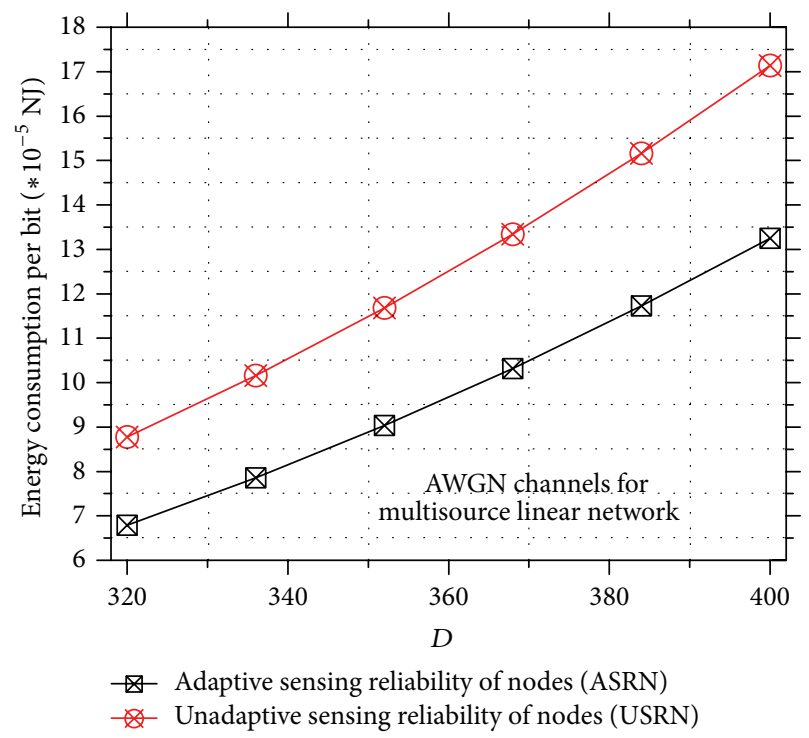

FIGURE 14: The energy consumption under ASRN and USRN.

TABLE 3: The reliability of node in multisource linear network (AWGN channels).

\begin{tabular}{lcccccccc}
\hline$c_{1}$ & $c_{2}$ & $c_{3}$ & $c_{4}$ & $c_{5}$ & $c_{6}$ & $c_{7}$ & $c_{8}$ & $C$ \\
\hline 0.9 & 0.92 & 1 & 1 & 1 & 1 & 1 & 1 & 0.828 \\
0.977 & 0.977 & 0.977 & 0.977 & 0.977 & 0.977 & 0.977 & 0.977 & 0.828 \\
\hline
\end{tabular}

the maximum energy consumption is higher than ASRN. Figure 14 shows the energy consumption under these two schemes.

5.2. Grid Network. This section is the verification of optimization design for a $5 * 5$ grid network.

Table 4 gives the node deployment distance with ASTDN in AWGN channels for grid network. Compared with linear network, since the node data load is more uneven in grid network, the node distance is more uneven. 
TABLE 4: The unequal transmission distance of nodes (AWGN channels).

\begin{tabular}{ccccccc}
\hline$d_{1}$ & $d_{2}$ & $d_{3}$ & $d_{4}$ & $d_{5}$ & $d_{6}$ & Total nodes \\
\hline 30 & 40 & 45 & 54 & 72 & 241 & $5 * 5$ \\
31 & 41 & 47 & 56 & 74 & 249 & $5 * 5$ \\
32 & 42 & 48 & 57 & 77 & 256 & $5 * 5$ \\
33 & 44 & 50 & 59 & 79 & 265 & $5 * 5$ \\
34 & 45 & 51 & 61 & 81 & 272 & $5 * 5$ \\
35 & 46 & 52 & 63 & 84 & 280 & $5 * 5$ \\
36 & 47 & 54 & 64 & 86 & 287 & $5 * 5$ \\
37 & 49 & 55 & 66 & 88 & 295 & $5 * 5$ \\
38 & 50 & 57 & 68 & 91 & 304 & $5 * 5$ \\
39 & 51 & 58 & 70 & 93 & 311 & $5 * 5$ \\
40 & 53 & 60 & 71 & 95 & 319 & $5 * 5$ \\
\hline
\end{tabular}

TABLE 5: The reliability of node in grid network (AWGN channels).

\begin{tabular}{lccccc}
\hline$c_{1}$ & $c_{2}$ & $c_{3}$ & $c_{4}$ & $c_{5}$ & $C$ \\
\hline 0.9 & 1 & 1 & 1 & 1 & 0.9 \\
0.97915 & 0.97915 & 0.97915 & 0.97915 & 0.97915 & 0.9 \\
\hline
\end{tabular}

Figure 15 gives the energy consumption under ASTDN and USTDN in AWGN channels. Compared with Figure 16, we can see that the energy consumption is reduced by about 4 times with USTDN over ASTDN in grid network.

Table 5 shows the nodes reliability with ASRN in AWGN channels. Since the node energy consumption is more uneven, the reliability of the first node is 0.9 ; the reliability of other nodes can be ensured as 1, while, under USRN, the reliability of each node should be 0.97915 to achieve network total reliability as 09 . Figure 17 gives the maximum energy consumption under ASRN and USRN; as can be seen, ASRN has better performance.

\section{Conclusion}

In this paper, we propose an adaptive sensing range optimal approach which not only achieves an optimal network lifetime but also guarantees the end-to-end reliability. We give the optimization for single-source linear network, multisource linear network, and grid network under additive white Gaussian noise channels; the main contributions are as follows.

(1) We conduct theoretical analysis to obtain the optimal node number $N^{*}$, node placement $d^{*}$, and node transmission structure $P^{*}$ which can achieve minimum total energy consumption and minimum unit data transmitting energy. We find that when the network total energy consumption for unit data is the minimum, the unit data energy consumption $\xi$ is not necessarily the minimum, while if $\xi$ is minimized, the network utilization is maximized.

(2) The minimum $\xi$ does not necessarily maximize utilization efficiency $\eta$. We observe that nodes near the sink have largest energy consumption, which

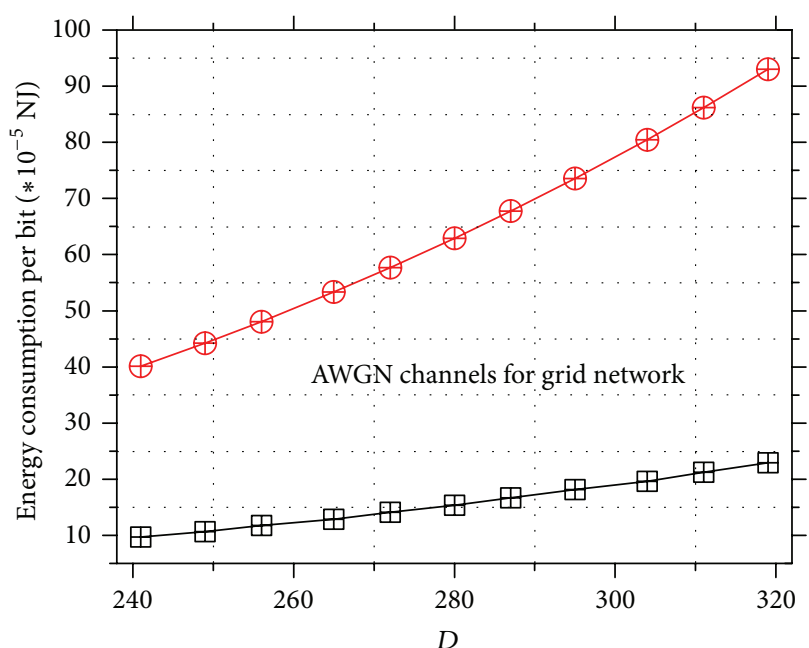

- - Adaptive sensing transmission distance of nodes (ASTDN)

(1)-Unadaptive sensing transmission distance of nodes (USTDN)

FIGURE 15: The energy consumption under ASTDN and USTDN.

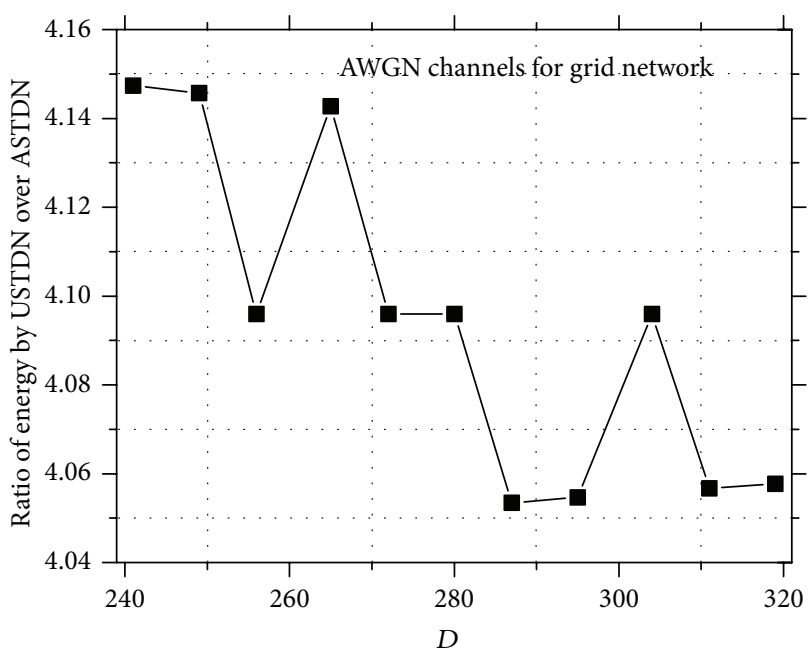

FIGURE 16: Ratio of energy by USTDN over ASTDN.

determines the network lifetime. Therefore, we propose an adaptive sensing range optimal design which adopts lower node reliability and smaller transmission distance for nodes near the sink; meanwhile, for nodes far from the sink, we adopt higher node reliability and bigger transmission distance. Through our design, the network energy consumption can be balanced, and thus the network utilization is improved. Besides, we give solvability conditions strictly from mathematics. We find that the network lifetime can be improved by several times (1-5 times) with ASTDN, compared with USTDN. Meanwhile, the network lifetime can be improved by more than $25 \%$ with ASRN compared with USRN.

(3) Different from previous research, this paper strictly from mathematics gives the optimization equations 


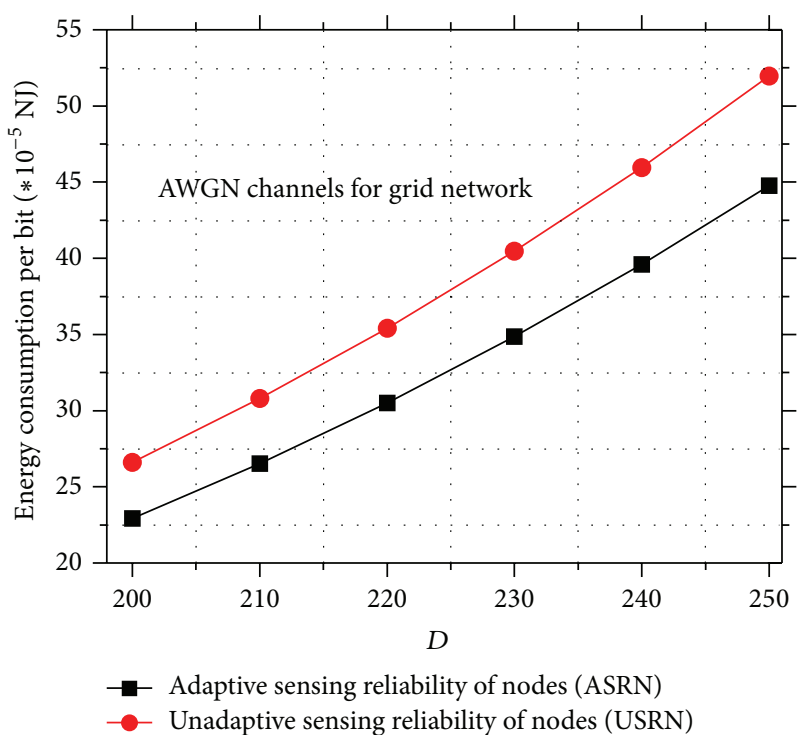

FIGURE 17: The energy consumption under ASRN and USRN.

and their solvable conditions, which has good theoretical significance.

\section{Conflict of Interests}

The authors declare that there is no conflict of interests regarding the publication of this paper.

\section{Acknowledgments}

This work was supported in part by the National Natural Science Foundation of China (61272150, 61379110, and 61472450), Ministry Education Foundation of China (20130162110079 and MCM20121031), the National High Technology Research and Development Program of China (863 Program) (2012AA010105), the National Basic Research Program of China (973 Program) (2014CB046305), the Hunan Province Education Science Project (XJK015CXX006), the Natural Science Foundation of Hunan Provincial (13JJ4087), and Central South University of Forestry and Technology Youth Fund Project (QJ2011010B).

\section{References}

[1] S. H. Chauhdary, J. Lee, S. C. Shah, and M.-S. Park, "EBCOefficient boundary detection and tracking continuous objects in WSNs," KSII Transactions on Internet and Information Systems, vol. 6, no. 11, pp. 2901-2919, 2012.

[2] S. He, X. Gong, J. Zhang, J. Chen, and Y. Sun, "Curve-based deployment for barrier coverage in wireless sensor networks," IEEE Transactions on Wireless Communications, vol. 13, no. 2, pp. 724-735, 2014.

[3] J. Li and J. Cao, "Survey of object tracking in wireless sensor networks," Ad Hoc \& Sensor Wireless Networks, vol. 25, no. 1-2, pp. 89-120, 2015.

[4] S. He, J. Chen, X. Li, X. S. Shen, and Y. Sun, "Mobility and intruder prior information improving the barrier coverage of sparse sensor networks," IEEE Transactions on Mobile Computing, vol. 13, no. 6, pp. 1268-1282, 2014.

[5] S.-J. Kim and D.-J. Park, "A self-calibrated localization system using chirp spread spectrum in a wireless sensor network," KSII Transactions on Internet and Information Systems, vol. 7, no. 2, pp. 253-270, 2013.

[6] D. Miorandi, S. Sicari, F. De Pellegrini, and I. Chlamtac, "Internet of things: vision, applications and research challenges," $\mathrm{Ad}$ Hoc Networks, vol. 10, no. 7, pp. 1497-1516, 2012.

[7] S. He, J. Chen, F. Jiang, D. K. Y. Yau, G. Xing, and Y. Sun, "Energy provisioning in wireless rechargeable sensor networks," IEEE Transactions on Mobile Computing, vol. 12, no. 10, pp. 1931-1942, 2013.

[8] J. Long, A. Liu, M. Dong, and Z. Li, "An energy-efficient and sink-location privacy enhanced scheme for WSNs through ring based routing," Journal of Parallel and Distributed Computing, vol. 81-82, pp. 47-65, 2015.

[9] J. Li, X. Jiang, and I.-T. Lu, "Energy balance routing algorithm based on virtual MIMO scheme for wireless sensor networks," Journal of Sensors, vol. 2014, Article ID 589249, 8 pages, 2014.

[10] A. Liu, Z. Liu, M. Nurudeen, X. Jin, and Z. Chen, "An elaborate chronological and spatial analysis of energy hole for wireless sensor networks," Computer Standards and Interfaces, vol. 35, no. 1, pp. 132-149, 2013.

[11] P. Cheng, S. He, F. Jiang, Y. Gu, and J. Chen, "Optimal scheduling for quality of monitoring in wireless rechargeable sensor networks," IEEE Transactions on Wireless Communications, vol. 12, no. 6, pp. 3072-3084, 2013.

[12] L. Jun, M. Dong, K. Ota, and A. Liu, "Achieving source location privacy and network life-time maximization through treebased diversionary routing in WSNs," IEEE Access, vol. 2, pp. 633-651, 2014.

[13] L. A. Villas, A. Boukerche, H. S. Ramos, H. A. de Oliveira, R. B. de Araujo, and A. A. Loureiro, "DRINA: a lightweight and reliable routing approach for in-network aggregation in wireless sensor networks," IEEE Transactions on Computers, vol. 62, no. 4, pp. 676-689, 2013.

[14] A. Liu, D. Zhang, P. Zhang, G. Cui, and Z. Chen, "On mitigating hotspots to maximize network lifetime in multi-hop wireless sensor network with guaranteed transport delay and reliability," Peer-to-Peer Networking and Applications, vol. 7, no. 3, pp. 255273, 2014.

[15] Y. Li, Y. Shen, and K. Chi, "A lifetime-preserving and delayconstrained data gathering tree for unreliable sensor networks," KSII Transactions on Internet and Information Systems, vol. 6, no. 12, pp. 3219-3236, 2012.

[16] T. K. Jain, D. S. Saini, and S. V. Bhooshan, "Lifetime optimization of a multiple sink wireless sensor network through energy balancing," Journal of Sensors, vol. 2015, Article ID 921250, 6 pages, 2015.

[17] J. Liang and T. Li, "A maximum lifetime algorithm for data gathering without aggregation in wireless sensor networks," Applied Mathematics \& Information Sciences, vol. 7, no. 5, pp. 1705-1719, 2013.

[18] J. He, L. Duan, F. Hou, P. Cheng, and J. Chen, "Multiperiod scheduling for wireless sensor networks: a distributed consensus approach," IEEE Transactions on Signal Processing, vol. 63, no. 7, pp. 1651-1663, 2015.

[19] L. Jiang, A. Liu, Y. Hu, and Z. Chen, "Lifetime maximization through dynamic ring-based routing scheme for correlated data collecting in WSNs," Computers \& Electrical Engineering, vol. 41, no. 1, pp. 191-215, 2015. 
[20] J. Liang, J. Wang, X. Zhang, and J. Chen, "An adaptive probability broadcast-based data preservation protocol in wireless sensor networks," in Proceedings of the IEEE International Conference on Communications (ICC'11), June 2011.

[21] R. Zhang, O. Berder, J.-M. Gorce, and O. Sentieys, "Energydelay tradeoff in wireless multihop networks with unreliable links," Ad Hoc Networks, vol. 10, no. 7, pp. 1306-1321, 2012.

[22] P. Chen, B. O'Dea, and E. Callaway, "Energy efficient system design with optimum transmission range for wireless ad hoc networks," in Proceedings of the International Conference on Communications (ICC '02), vol. 2, pp. 945-952, May 2002.

[23] J. L. Gao, "Analysis of energy consumption for ad hoc wireless sensor networks using a bit-meter-per-joule metric," IPN Progress Report 42-150, 2002.

[24] Y. Chen, C.-N. Chuah, and Q. Zhao, "Network configuration for optimal utilization efficiency of wireless sensor networks," Ad Hoc Networks, vol. 6, no. 1, pp. 92-107, 2008.

[25] M. Tahir and R. Farrell, "A cross-layer framework for optimal delay-margin, network lifetime and utility tradeoff in wireless visual sensor networks," Ad Hoc Networks, vol. 11, no. 2, pp. 701711, 2013.

[26] Y. Liu, A. Liu, and Z. Chen, "Analysis and improvement of send-and-wait automatic repeat-reQuest protocols for wireless sensor networks," Wireless Personal Communications, vol. 81, no. 3, pp. 923-959, 2015.

[27] S. Sastry, T. Radeva, J. Chen, and J. L. Welch, "Reliable networks with unreliable sensors," Pervasive and Mobile Computing, vol. 9, no. 2, pp. 311-323, 2013.

[28] A. Liu, X. Jin, G. Cui, and Z. Chen, "Deployment guidelines for achieving maximum lifetime and avoiding energy holes in sensor network," Information Sciences, vol. 230, pp. 197-226, 2013. 

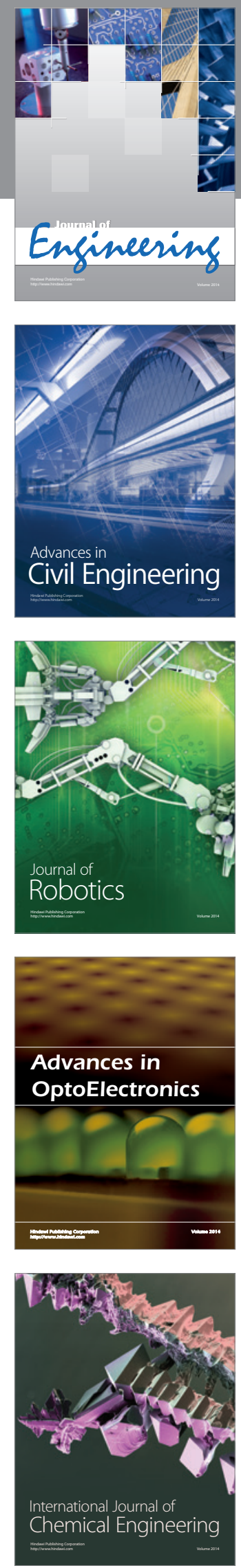

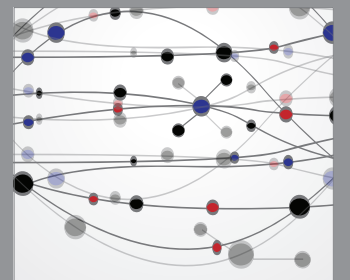

The Scientific World Journal
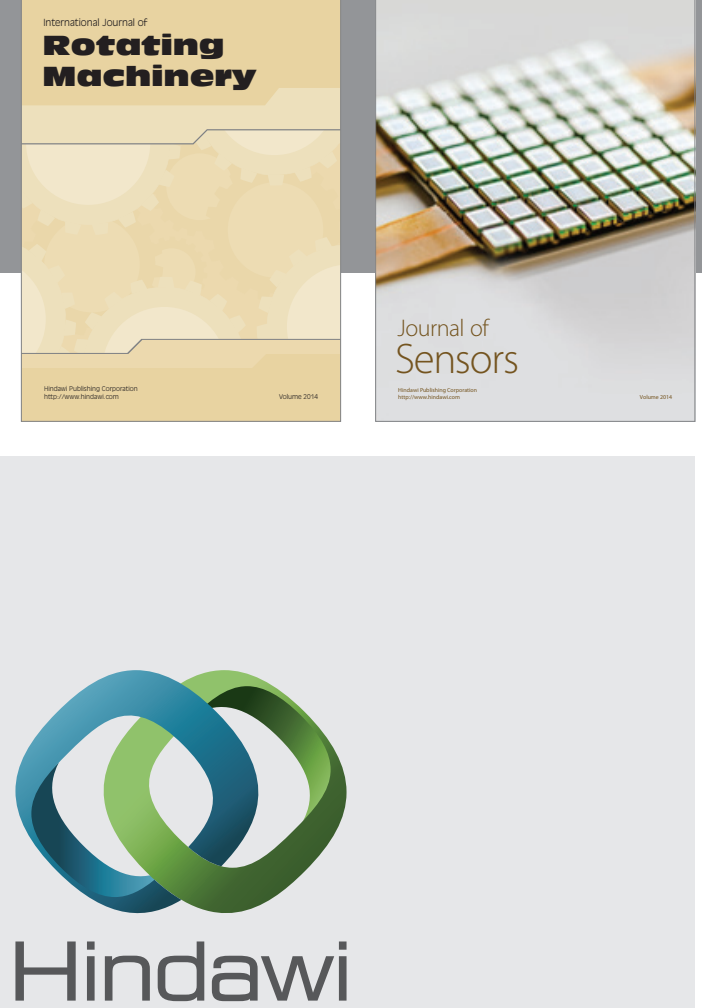

Submit your manuscripts at http://www.hindawi.com
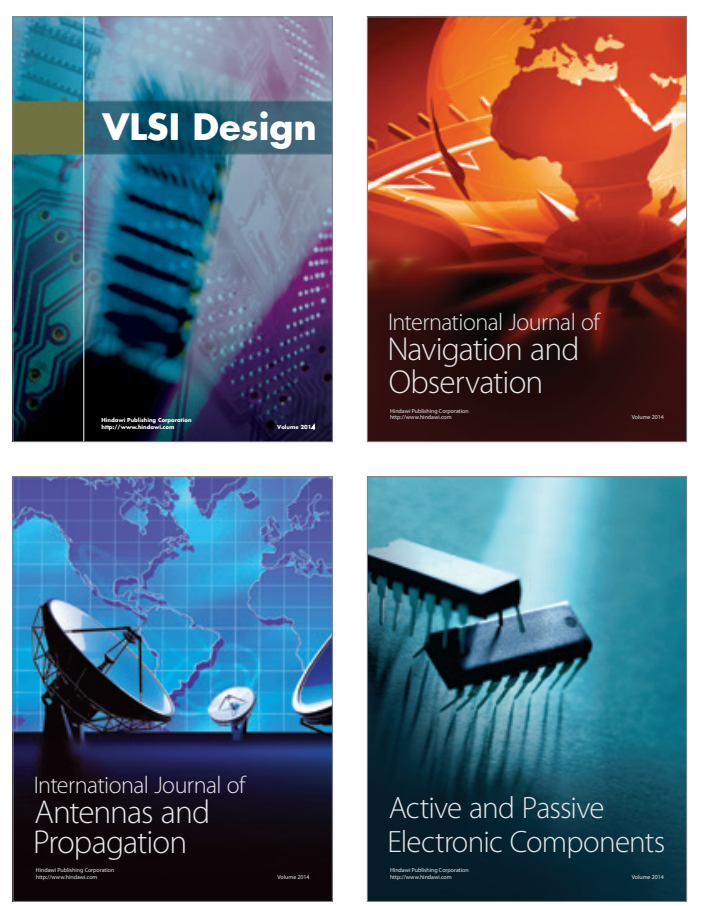
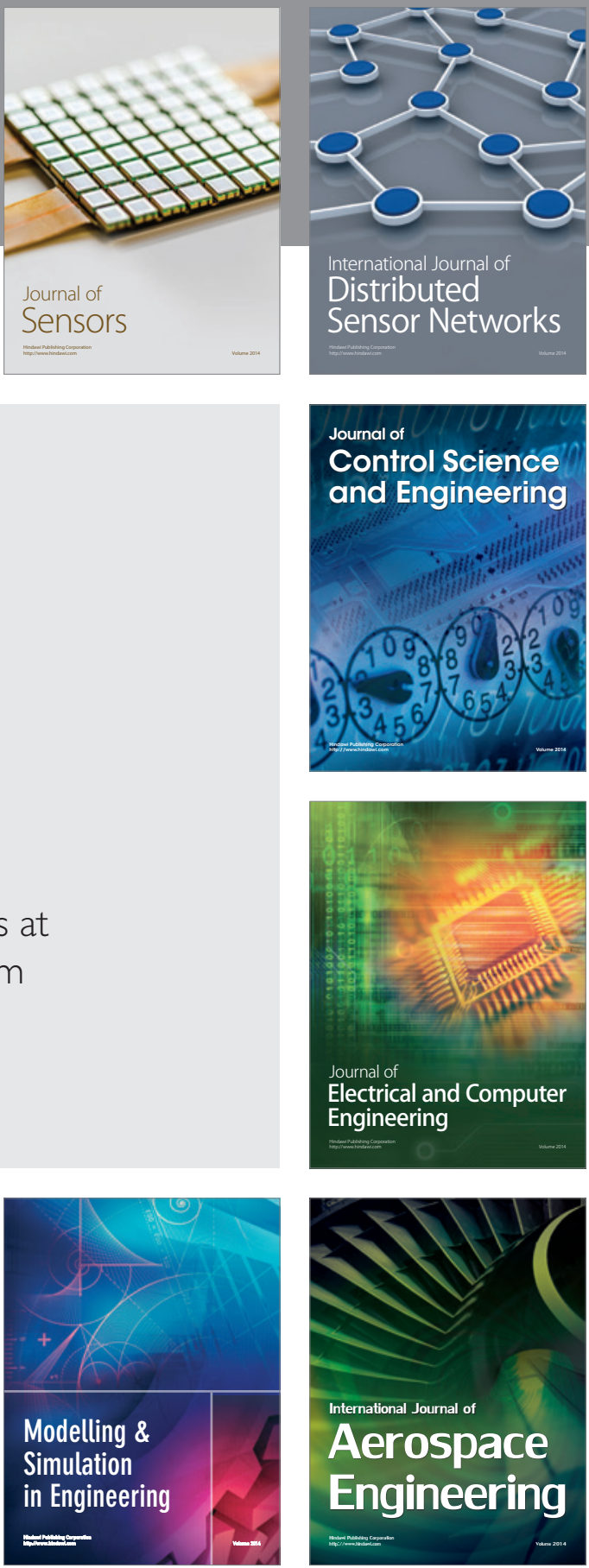

Journal of

Control Science

and Engineering
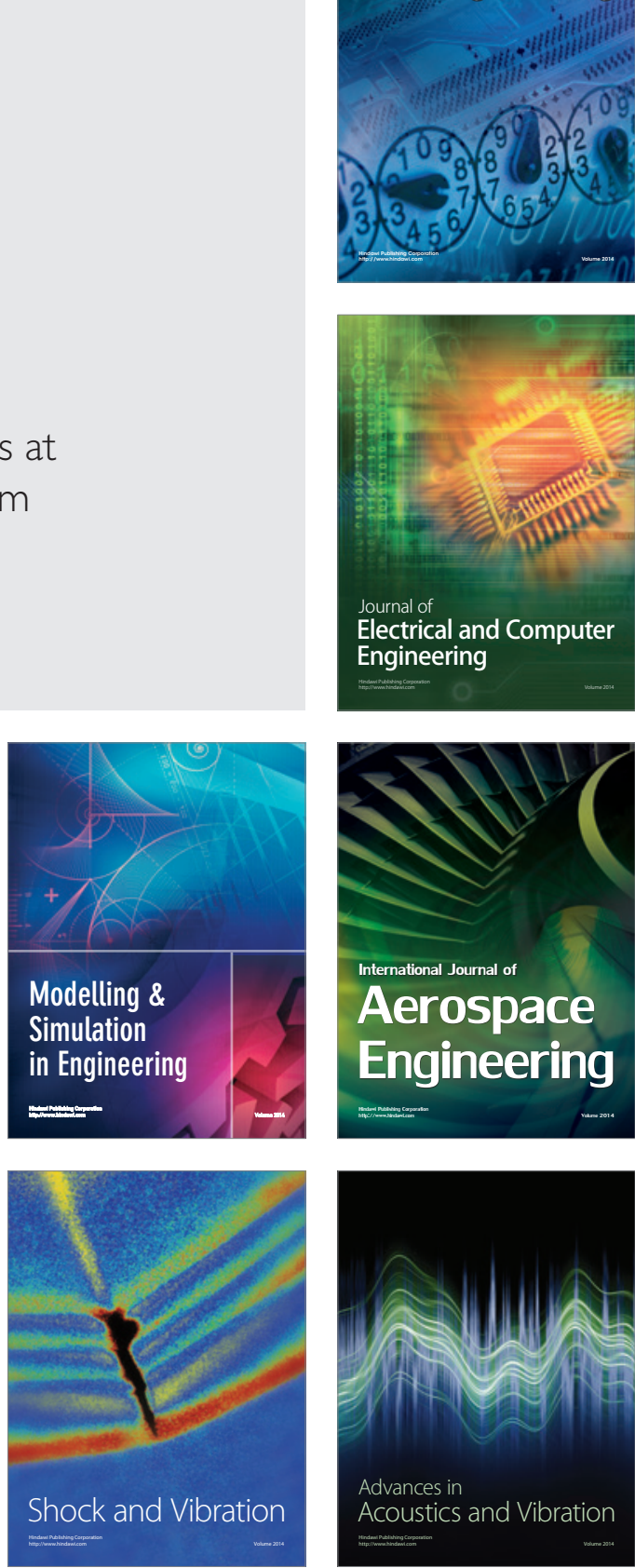